ARTICLE

DOI: $10.1038 / \mathrm{s} 41467-017-02494-0$

\title{
Dysbindin links presynaptic proteasome function to homeostatic recruitment of low release probability vesicles
}

\author{
Corinna Wentzel', Igor Delvendahl', Sebastian Sydlik¹,2, Oleg Georgiev ${ }^{1}$ \& Martin Müller ${ }^{1,2,3}$
}

\begin{abstract}
Here we explore the relationship between presynaptic homeostatic plasticity and proteasome function at the Drosophila neuromuscular junction. First, we demonstrate that the induction of homeostatic plasticity is blocked after presynaptic proteasome perturbation. Proteasome inhibition potentiates release under baseline conditions but not during homeostatic plasticity, suggesting that proteasomal degradation and homeostatic plasticity modulate a common pool of vesicles. The vesicles that are regulated by proteasome function and recruited during homeostatic plasticity are highly EGTA sensitive, implying looser $\mathrm{Ca}^{2+}$ influx-release coupling. Similar to homeostatic plasticity, proteasome perturbation enhances presynaptic $\mathrm{Ca}^{2+}$ influx, readily-releasable vesicle pool size, and does not potentiate release after loss of specific homeostatic plasticity genes, including the schizophrenia-susceptibility gene dysbindin. Finally, we provide genetic evidence that Dysbindin levels regulate the access to EGTA-sensitive vesicles. Together, our data suggest that presynaptic protein degradation opposes the release of low-release probability vesicles that are potentiated during homeostatic plasticity and whose access is controlled by dysbindin.
\end{abstract}

\footnotetext{
${ }^{1}$ Institute of Molecular Life Sciences, University of Zurich, Winterthurerstrasse 190, 8057 Zurich, Switzerland. ${ }^{2}$ Zurich Ph.D. Program in Molecular Life Sciences, Winterthurerstrasse 190, Zurich, 8057, Switzerland. ${ }^{3}$ Neuroscience Center Zurich, Winterthurerstrasse 190, Zurich, 8057, Switzerland. Correspondence and requests for materials should be addressed to M.Mül. (email: Martin.Mueller@imls.uzh.ch)
} 
$\mathrm{M}$ ost synapses function robustly within neural circuits throughout the lifetime of an animal. However, the proteins determining synaptic activity are turned over on relatively rapid time scales of hours to weeks ${ }^{1-3}$. Thus, individual synapses are continuously 'rebuilding' themselves. It is therefore remarkable that robust neural function can be maintained at all. Work during the last decades has revealed that synaptic transmission is actively stabilized by evolutionarily conserved homeostatic signaling systems, but the underlying molecular mechanisms are largely unknown ${ }^{4-7}$.

The Drosophila neuromuscular junction (NMJ) has emerged as a powerful model synapse (note that we use 'NMJ' and 'synapse' interchangeably) to unravel the genetic basis of presynaptic homeostatic plasticity $(\mathrm{PHP})^{8}$. At this synapse, postsynaptic glutamate receptor perturbation induces a homeostatic increase in neurotransmitter release that precisely restores postsynaptic depolarization in response to action-potential (AP) stimulation $^{9,10}$. An electrophysiology-based genetic screen has implicated several genes in this form of synaptic plasticity ${ }^{11-18}$. All of the identified genes are evolutionarily conserved to humans, and lossof-function mutations in these genes block PHP. Nevertheless, it has remained unclear how most of the identified genes participate in homeostatic signaling.

One of the genes that is required for PHP in Drosophila is dysbindin ${ }^{11}$, a gene linked to schizophrenia in humans $\left(D T N B P 1^{19,20}\right)$. There is genetic evidence that dysbindin functions in concert with snapin and SNAP-25 during PHP ${ }^{21}$. Recent genetic data suggest that $d y$ sbindin-dependent homeostatic regulation of release involves the BLOC-1 complex member Blos $1^{22}$ and the Arp2/3 actin polymerization complex ${ }^{23}$. Moreover, the role of dysbindin in PHP appears to be independent of presynaptic $\mathrm{Ca}^{2+}$ influx or major changes in synapse development ${ }^{11}$. However, besides these genetic data little is known about how dysbindin is involved in PHP.

At the Drosophila NMJ, PHP can be rapidly induced within minutes in the presence of the protein synthesis inhibitor cyclohexamide ${ }^{12}$, suggesting that the acute induction of PHP does not require synthesis of new proteins. In contrast, protein degradation has not been studied in the context of PHP at this synapse. The ubiquitin-proteasome system (UPS) is a major protein degradation pathway ${ }^{24}$. At the Drosophila NMJ it has been shown that all components of the UPS are present at presynaptic terminals and that acute proteasome inhibition causes a rapid strengthening of neurotransmission ${ }^{3}$. There is accumulating evidence for links between neural activity and UPS-mediated degradation of presynaptic proteins in mice and rats $^{25-30}$. However, only two presynaptic proteins-Rab3-interacting molecule ( $\mathrm{RIM}^{25,26,30}$ ) and Dunc-13/munc-13 ${ }^{3}$ - as well as one E3-ligase (SCRAPPER ${ }^{30}$ ) have so far been implicated in UPS-dependent control of presynaptic protein turnover and release. Thus, the molecular pathways underlying the regulation of presynaptic release through protein degradation remain enigmatic.

PHP at the Drosophila NMJ requires high-release probability $\left(p_{r}\right)$ vesicles that are 'tightly coupled' to $\mathrm{Ca}^{2+}$ channels through rimbinding protein ${ }^{16}$. The distance between $\mathrm{Ca}^{2+}$ channels and $\mathrm{Ca}^{2+}$ sensors of exocytosis, typically referred to as 'coupling distance', is a major factor determining the $p_{r}$ of synaptic vesicles ${ }^{31-34}$. However, despite their implication in short-term plasticity, little is known about how vesicles that are differentially coupled to $\mathrm{Ca}^{2+}$ influx are modulated during synaptic plasticity.

Here we explore the relationship between PHP and presynaptic proteasome function at the Drosophila NMJ and provide evidence for links between presynaptic protein degradation and homeostatic potentiation of loosely-coupled synaptic vesicles.

\section{Results}

PHP requires presynaptic proteasome function. To investigate potential links between protein degradation and PHP induced by postsynaptic glutamate receptor perturbation, we probed the effects of acute pharmacological proteasome inhibition on PHP at the Drosophila NMJ (Fig. 1). First, we acutely perturbed proteasome function by application of the proteasome inhibitor lactacystin ( $100 \mu \mathrm{M}$ for $15 \mathrm{~min}$; Supplementary Fig. 1), which has been demonstrated to interfere with synaptic proteasome function at the Drosophila NMJ before ${ }^{3}$. Using this manipulation, we asked if protein degradation is required for the acute induction of PHP in response to pharmacological glutamate receptor perturbation ${ }^{12}$. Application of sub-saturating concentrations of the glutamate receptor antagonist philanthotoxin-433 (PhTX, $20 \mu \mathrm{M}$ for 10 $\mathrm{min}$ ) led to a significant decrease in miniature excitatory postsynaptic potential (mEPSP) amplitude at both control and lactacystin-treated synapses (Fig. 1b, left). At control synapses, the decrease in mEPSP amplitude in the presence of PhTX was accompanied by a significant increase in neurotransmitter release ('quantal content'=EPSP amplitude/mEPSP amplitude; Fig. 1b, c, right), thereby restoring AP-evoked EPSP amplitudes to baseline levels (Fig. 1b, middle), as expected from previous work ${ }^{9,12}$. By contrast, synapses that had previously been incubated with lactacystin had elevated baseline EPSP amplitudes (Fig. 1b, middle; see below), and PHP was completely blocked, as shown by similar quantal content values for lactacystin-treated synapses in the absence and presence of PhTX (Fig. 1b, right). As a consequence, EPSP amplitudes were significantly decreased after PhTX treatment (Fig. 1b, middle), to a similar degree as mEPSP amplitudes (Fig. 1b, left). Similar results were obtained with the proteasome inhibitor MG-132 (Fig. 1b). These data demonstrate that proteasome function is required for the acute induction of PHP.

We also observed that lactacystin treatment potentiates quantal content (Fig. 1b, right) in a concentration-dependent and incubation time-dependent manner under baseline conditions without affecting mEPSP frequency (Supplementary Fig. 1, 2a and b), consistent with previous work ${ }^{3}$. The block of PHP after lactacystin treatment could be due to saturation of quantal content. Although we cannot fully exclude a ceiling effect, we consider this unlikely because release was not saturated after lactacystin application under our recording conditions (quantal content $\sim 35$ vs. $\sim 50$ after PhTX treatment; Fig. 1b), and because PHP was completely blocked after proteasome perturbation at elevated extracellular $\mathrm{Ca}^{2+}$ concentration $(1 \mathrm{mM}$; see below). Together, our results suggest that proteasome function opposes the release of synaptic vesicles that are required for PHP.

The effects of lactacystin could arise from presynaptic and/or postsynaptic proteasome impairment. To distinguish between these two possibilities, we took advantage of the Gal4/UAS expression system to specifically perturb either presynaptic or postsynaptic proteasome function (neuronal driver line elav $v^{c 155}$ Gal4, and muscle-specific 24B-Gal4 driver line, respectively). Using these Gal4 lines, we overexpressed one or two dominant temperature-sensitive mutant proteasome subunits (UAS-DTS5 and UAS-DTS7; see Methods), which were demonstrated to impair proteasome function in Drosophila because of mutations in $\beta$-subunits of the $20 \mathrm{~S}$ proteasome ${ }^{35}$. Under our experimental conditions (expression at the permissive temperature, $25^{\circ} \mathrm{C}$, throughout development), DTS expression is thought to result in a hypomorphic phenotype, i.e., a perturbation, but not complete loss of proteasome function ${ }^{35}$. We first assessed if presynaptic overexpression of two DTS copies-henceforth referred to as 'DTS pre' - affects the acute induction of PHP upon PhTX treatment (Fig. 1a, c). Application of PhTX to DTS pre mutants 
a
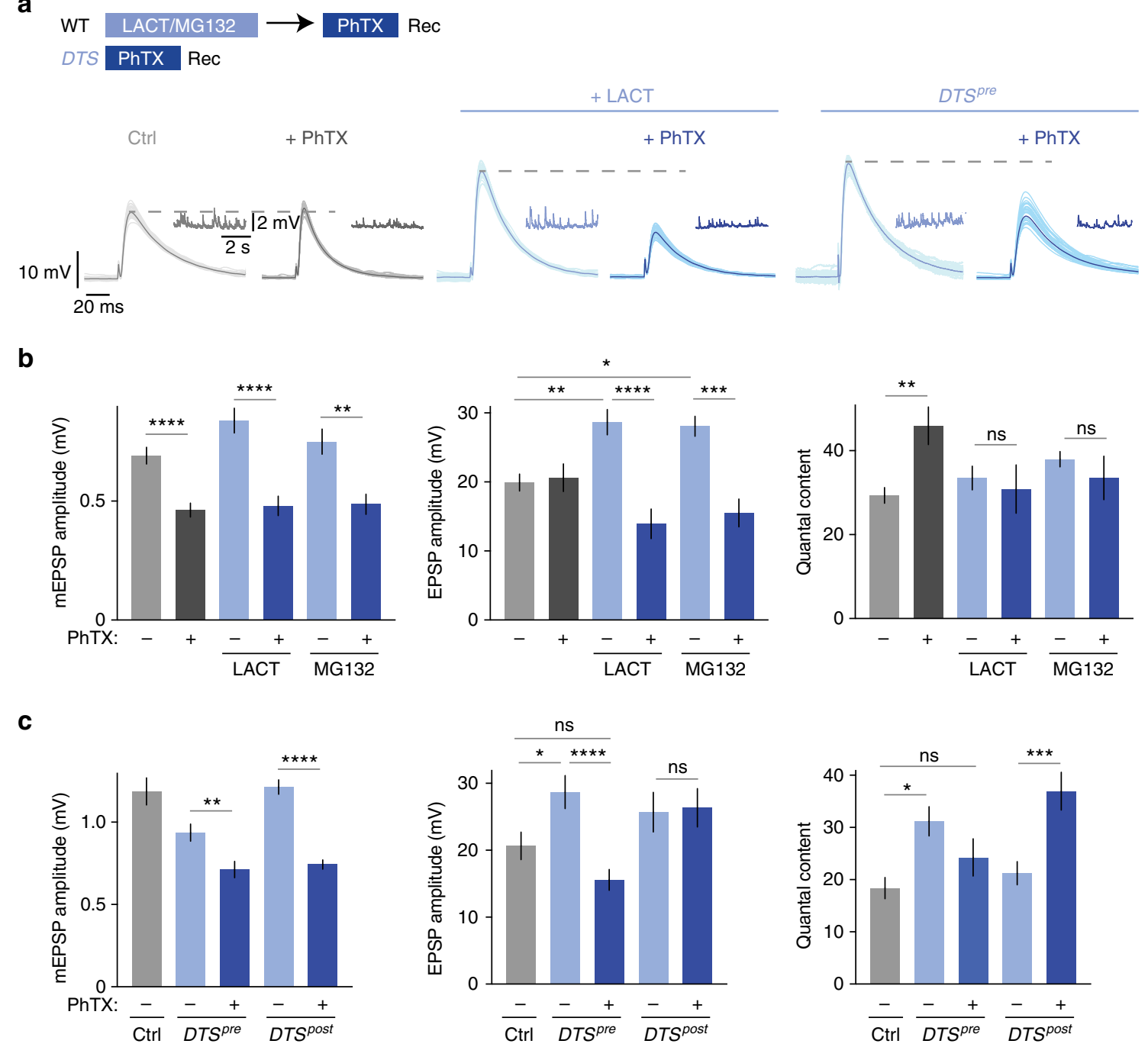

Fig. 1 Presynaptic proteasome perturbation blocks presynaptic homeostatic plasticity. a Scheme for treatment of NMJs and representative EPSP and mEPSP traces (Rec: recording). Wild-type NMJs were incubated with saline, $100 \mu \mathrm{M}$ lactacystin for 15 min or $50 \mu \mathrm{M}$ MG-132 for 15 min, followed by wash and treatment with saline or $20 \mu \mathrm{M} \mathrm{PhTX}$ for $10 \mathrm{~min}$ at $0.3 \mathrm{mM}\left[\mathrm{Ca}^{2+}\right]_{\mathrm{e}}$. DTS-mutant synapses were treated with saline or $20 \mu \mathrm{M} \mathrm{PhTX}$ for $10 \mathrm{~min}$ at 0.3 $\mathrm{mM}\left[\mathrm{Ca}^{2+}\right]_{\mathrm{e}}$. A total of 30 sweeps are shown per cell, with the average displayed in darker color. $\mathbf{b}$ Quantification of mEPSP amplitude, EPSP amplitude and quantal content for wild-type synapses treated as described in (a). PhTX treatment induced an increase in quantal content (right). Note that PhTX application after lactacystin or MG-132 treatment completely blocks the homeostatic increase in quantal content (right). Mean \pm s.e.m.; $n \geq 6$ cells; ${ }^{\star} p<$ $0.05 ;{ }^{\star \star} p<0.001 ;{ }^{\star \star \star} p<0.0001 ;{ }^{* \star \star \star} p<0.00001$; ns: not significant; ANOVA and Tukey's multiple comparison tests for comparison of EPSP amplitudes, Student's t-test for pairwise comparison of mEPSP amplitude and quantal content in PhTX-treated vs. non-PhTX-treated cells. c Quantification of mEPSP amplitude, EPSP amplitude and quantal content of control synapses (elav ${ }^{\mathrm{C} 155}$-Gal4 and 24B-Gal4) and synapses expressing mutant proteasome subunits presynaptically (elav ${ }^{C 155}$ _Gal4;UAS-DTS5/+;UAS-DTS7/+; 'DTS pre') or postsynaptically (UAS-DTS5/+;UAS-DTS7/24B-Gal4; 'DTSpost'). Synapses were treated with $20 \mu \mathrm{M}$ PhTX or saline for $10 \mathrm{~min}$ at $0.3 \mathrm{mM}\left[\mathrm{Ca}^{2+}\right]_{\mathrm{e}}$. Note that there is no increase in quantal content upon PhTX treatment after genetic presynaptic proteasome perturbation (DTS pre), indicating a defect in presynaptic homeostatic plasticity. PhTX treatment after postsynaptic proteasome perturbation potentiates quantal content (right), demonstrating that postsynaptic proteasome mutants do not have a defect in homeostatic plasticity. Mean \pm s.e.m.; $n \geq 10$ cells; ${ }^{\star} p<0.05 ;{ }^{\star \star} p<0.001 ;{ }^{\star \star \star} p<0.0001 ;{ }^{\star \star \star \star} p<0.00001$; ns: not significant; ANOVA and Tukey's multiple comparison tests

reduced mEPSP amplitudes (Fig. 1c, left), but failed to increase quantal content with respect to untreated DTS mutants (Fig. 1c, right), resulting in significantly smaller EPSP amplitudes compared to untreated mutants (Fig. 1c, middle). These data demonstrate that the presynaptic proteasome is required for PHP. We also detected a significant increase in EPSP amplitude and quantal content in DTS ${ }^{\text {pre }}$ mutants under baseline conditions (Fig. 1a, c) that was similar to the lactacystin-induced increase in release (Fig. 1b).

Moreover, while observing a significant increase in release after presynaptic expression of two DTS copies, there was no apparent change in synaptic transmission upon expressing one DTS copy
(Supplementary Fig. $2 c$ and d). This implies a dose-dependent effect of DTS expression on release, similar to lactacystin treatment (Supplementary Fig. 1). Again, release was not saturated at DTS ${ }^{\text {pre }}$-mutant synapses in the absence of PhTX (Fig. 1c), suggesting that presynaptic genetic proteasome inhibition unlikely occluded PHP, similar to pharmacological proteasome inhibition.

By contrast, PhTX treatment of postsynaptic DTS-mutant synapses ('DTS ${ }^{\text {post', }}$ Fig. 1c) enhanced quantal content with respect to untreated mutants (Fig. 1c, right), thereby restoring EPSP amplitudes to baseline levels (Fig. 1c, middle). Hence, postsynaptic proteasome function is not required for the acute 
induction of PHP, underlining the relevance of the presynaptic proteasome in this form of synaptic plasticity.

A recent study demonstrated reduced presynaptic proteasome number and mobility because of a defect in proteasome trafficking in mutants lacking cut-up $(c t p)^{36}$, a gene encoding the Drosophila homolog of the dynein light chain subunit LC8. Interestingly, we revealed that loss of ctp disrupts PHP (Supplementary Fig. 2e and f), further supporting our conclusion that presynaptic proteasome function is required for PHP.

In addition, we tested if the enhancement in synaptic strength observed after lactacystin application goes along with an increase in ubiquitinated protein levels at the synapse (Supplementary Fig. 1d-g). To assay synaptic levels of ubiquitin-tagged proteins, we utilized an antibody (FK2) against mono- and polyubiquitinated proteins but not free ubiquitin ${ }^{37}$. Lactacystin treatment induced a pronounced increase in FK2-fluorescence intensity throughout the synapse and the postsynaptic muscle cell (Supplementary Fig. 1d-g). Since our physiology data suggest a presynaptic function, we quantified FK2-fluorescence intensity in the presynaptic compartment using a mask based on the neuronal membrane marker horseradish peroxidase (HRP, see Methods ${ }^{38}$ ) and detected a marked increase in FK2 fluorescence without apparent changes in HRP fluorescence upon lactacystin treatment (Supplementary Fig. 1e-g). These data demonstrate that the increase in neurotransmitter release is correlated with a rapid increase in presynaptic ubiquitinated proteins.

Proteasome perturbation does not enhance release during PHP. We demonstrated that lactacystin application enhances release under baseline conditions, whereas subsequent PhTX treatment fails to induce PHP. We next reversed the order of the experimental perturbations and probed the effects of lactacystin treatment on release after inducing PHP by PhTX incubation (Fig. 2a). An increase in release upon proteasome impairment after the induction of PHP would imply that both manipulations activate different cellular pathways resulting in additive effects. By contrast, an absence of an effect would indicate that both perturbations are non-additive. As shown above (Fig. 1), lactacystin treatment significantly increased EPSP amplitudes and quantal content at control synapses that were not treated with PhTX (Fig. 2a, b). However, we did not detect changes in EPSP amplitude or quantal content upon lactacystin treatment if synapses had been previously incubated with PhTX as compared to synapses that were treated with PhTX alone (Fig. 2a, b, middle, right). These results are consistent with the idea that the increase in release induced by either PhTX incubation or lactacystin treatment employ similar cellular mechanisms and are nonadditive.

At the Drosophila NMJ, PHP can be either acutely induced by PhTX application or chronically expressed through genetic ablation of the glutamate receptor subunit GluRIIA (GluR$\left.I I A^{S P 16}\right)^{10}$. We next investigated the relationship between proteasome function and the sustained expression of PHP (Fig. 2a, b). Similar to wild-type synapses that were treated with PhTX (Fig. 2a, b), GluRIIA mutants did not display significant changes in EPSP amplitude or quantal content after lactacystin treatment compared to saline-treated GluRIIA mutants (Fig. 2b). This observation implies that the mechanisms underlying acute and sustained PHP expression are required to potentiate release upon acute proteasome impairment.

Next, we generated transgenic flies that are mutant for GluRIIA and overexpress DTS presynaptically ('GluRIIA, DTS ${ }^{\text {pre'; flies }}$ were kept at the permissive temperature throughout development) to investigate presynaptic proteasome function in the context of sustained PHP. Similar to lactacystin application, genetic presynaptic proteasome inhibition caused no apparent changes in EPSP amplitude or quantal content compared to GluRIIA mutants alone (Fig. 2c, d). This suggests that sustained PHP expression involves mechanisms that are required to potentiate release upon proteasome inhibition. By extension, the fact that pharmacological or genetic proteasome perturbation do not induce apparent changes in synaptic physiology after the induction or sustained expression of PHP suggests that the effects of proteasome impairment on release are specific to homeostatic plasticity.

Next, we tested if synapses that undergo PHP exhibit changes in ubiquitinated protein levels using the FK2 antibody. We did not detect significant changes in FK2-fluorescence intensity upon PhTX treatment (Supplementary Fig. 3), but there was a consistent and significant increase in FK2-fluorescence intensity in the presynaptic mask of GluRIIA-mutant synapses compared to wild-type controls (Fig. 2e). Even if we cannot rule out that the observed increase in FK2-fluorescence intensity at GluRIIAmutant synapses is due to increased protein expression, these data suggest that the sustained expression of PHP is correlated with increased synaptic levels of ubiquitin-tagged proteins. The observation of no apparent changes in FK2-fluorescence intensity after PhTX incubation suggests that we either cannot resolve potential differences, or that non-ubiquitinated protein levels may be altered during acute homeostatic plasticity. Alternatively, different mechanisms may underlie the acute induction and the sustained expression of PHP.

Unaltered synapse morphology after proteasome perturbation. The defect in PHP in presynaptic DTS mutants could be caused by impaired synapse development. Therefore, we investigated synaptic morphology in flies overexpressing two DTS copies ('DTS ${ }^{\text {pre' }}$ ) neuronally throughout development (Fig. 3). Immunostainings of NMJs using antibodies detecting neuronal membranes ('aHRP', anti-horseradish peroxidase), presynaptic active zones ('aBrp', anti-Bruchpilot) and the postsynaptic reticulum ('aDLG', anti-discs large) revealed no apparent changes in synapse size (synaptic span, HRP area and DLG area), active zone number (Brp puncta number), active zone density (Brp puncta/ HRP area and Brp puncta/DLG area), active zone area (Brp area) or Brp intensity between DTS ${ }^{\text {re }}$ mutants and controls (Fig. 3b, Supplementary Fig. 4). Moreover, there were no apparent differences in glutamate receptor field number, size or glutamate receptor levels between DTS ${ }^{\text {re }}$ mutants and wild types at confocal resolution (Supplementary Fig. 4). Thus, the disruption of PHP is unlikely a secondary consequence of impaired anatomical development or reduced active zone number at presynaptic DTSmutant NMJs.

Presynaptic proteasome perturbation potentiates EPSC charge. Next, we examined PHP after presynaptic DTS expression using two-electrode voltage-clamp (TEVC) analysis at increased $\left[\mathrm{Ca}^{2+}\right]_{\mathrm{e}}$ (Fig. 4; $1 \mathrm{mM}\left[\mathrm{Ca}^{2+}\right]_{e}$ ). PhTX treatment significantly decreased mEPSC amplitude and mEPSC charge at control and DTS pre mutant synapses (Fig. 4a, d). At control synapses, the PhTXinduced decrease in mEPSC amplitude or mEPSC charge resulted in a significant increase in quantal content (Fig. 4e, gray data), which restored EPSC amplitudes and EPSC charge towards baseline values (Fig. 4b, gray data). By contrast, PhTX application did not potentiate quantal content in DTS ${ }^{\text {re }}$ mutants (Fig. 4e, blue data), so that EPSC amplitude and EPSC charge remained significantly smaller than at untreated mutant synapses (Fig. 4b, blue data). Thus, presynaptic proteasome function is also required for PHP at elevated $\left[\mathrm{Ca}^{2+}\right]_{\mathrm{e}}$. 
a

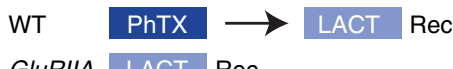

GIURIIA LACT Rec

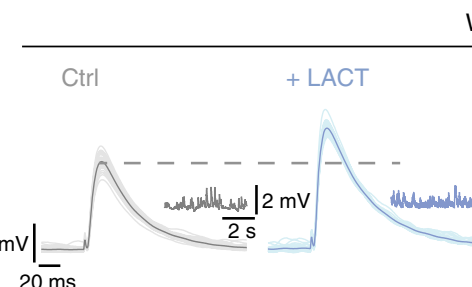

WT

+ PhTX $\quad+$ PhTX + LACT

\begin{tabular}{ll} 
GluRIIA \\
\hline Ctrl \\
+ LACT
\end{tabular}
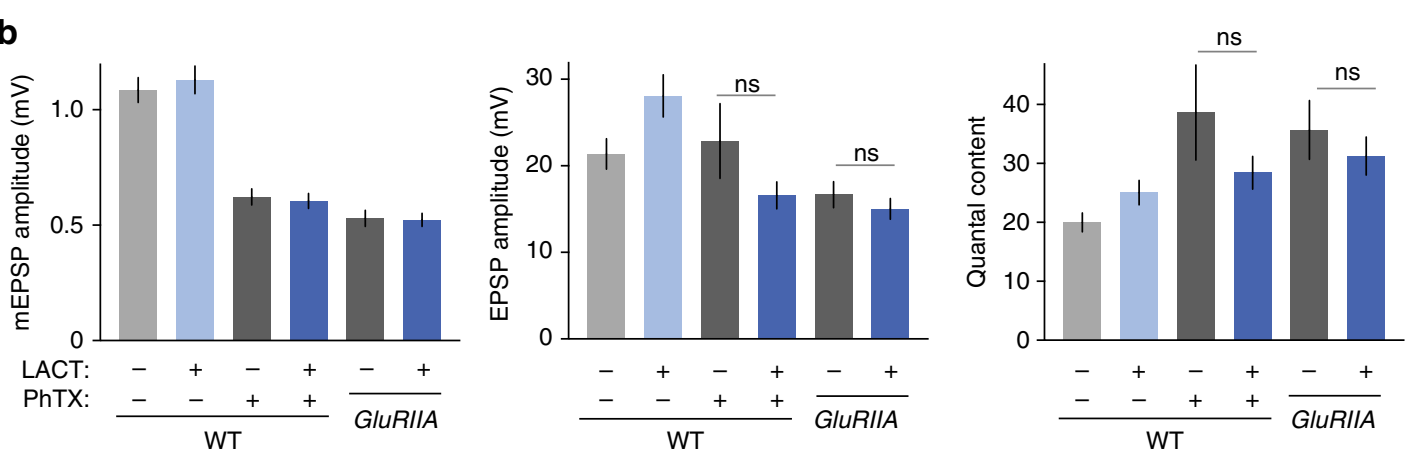

C
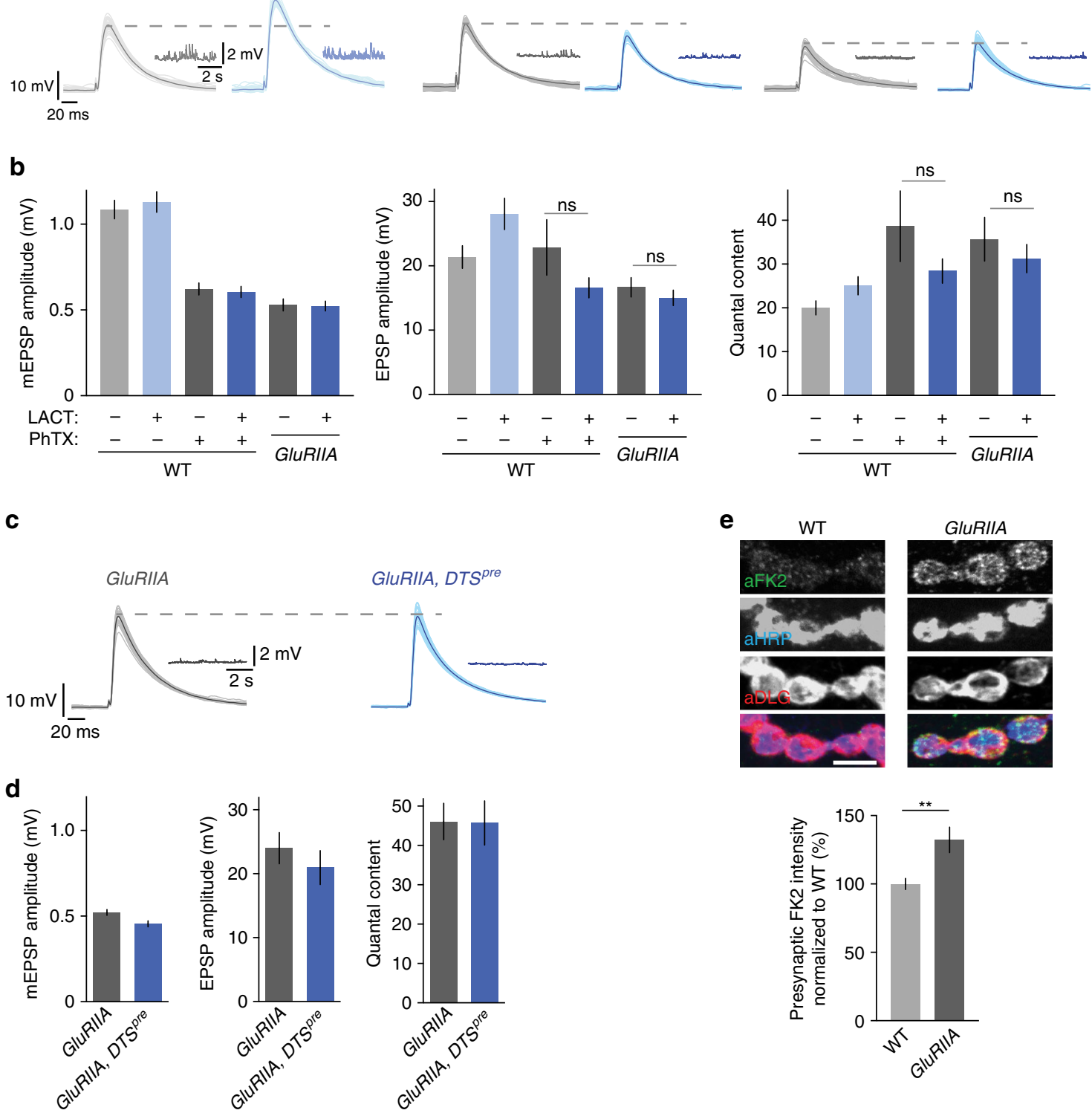

Fig. 2 Proteasome perturbation does not potentiate release after acute induction or sustained expression of PHP. a Scheme for treatment of NMJs and representative EPSP and mEPSP traces. Wild-type NMJs were incubated with saline or $20 \mu \mathrm{M}$ PhTX for 10 min, followed by a wash and treatment with saline or $100 \mu \mathrm{M}$ lactacystin for $30 \mathrm{~min}$ at $0.3 \mathrm{mM}\left[\mathrm{Ca}^{2+}\right]_{\mathrm{e}}$. GluRIIA-mutant synapses were treated with saline or $100 \mu \mathrm{M}$ lactacystin for $30 \mathrm{~min}$ at $0.3 \mathrm{mM}$ $\left[\mathrm{Ca}^{2+}\right]_{\mathrm{e}}$. b Quantification of EPSP amplitude, mEPSP amplitude and quantal content for larvae treated as described in (a). Lactacystin application to larvae treated with PhTX or to GluRIIA-mutant larvae has no effect on EPSP amplitude or quantal content. Mean \pm s.e.m.; $n \geq 10$ cells; ns: not significant; Student's t-test. c Representative EPSP and mEPSP traces of GluRIIA-mutant synapses and GluRIIA,DTSpre double-mutant synapses. d Quantification of mEPSP amplitude, EPSP amplitude and quantal content of the genotypes described in (c). Presynaptic proteasome perturbation does not increase EPSP amplitude or quantal content in GluRIIA-mutant synapses. Mean \pm s.e.m.; $n \geq 7$ cells. e) Representative NMJs stained for mono- and polyubiquitinated proteins (aFK2), neuronal membrane (aHRP, horseradish peroxidase), and postsynaptic reticulum (aDLG, discs large) of wild-type and GluRIIA-mutant larvae (scale bar, $5 \mu \mathrm{m}$ ). Quantification of FK2-fluorescence intensity in the HRP mask (presynapse) suggests that GluRIIA-mutant NMJs have higher levels of mono- and polyubiquitinated proteins compared to wild-type NMJs. Mean \pm s.e.m.; $n=24$ NMJs; ${ }^{* *} p<0.001$; Student's $t$-test 
a
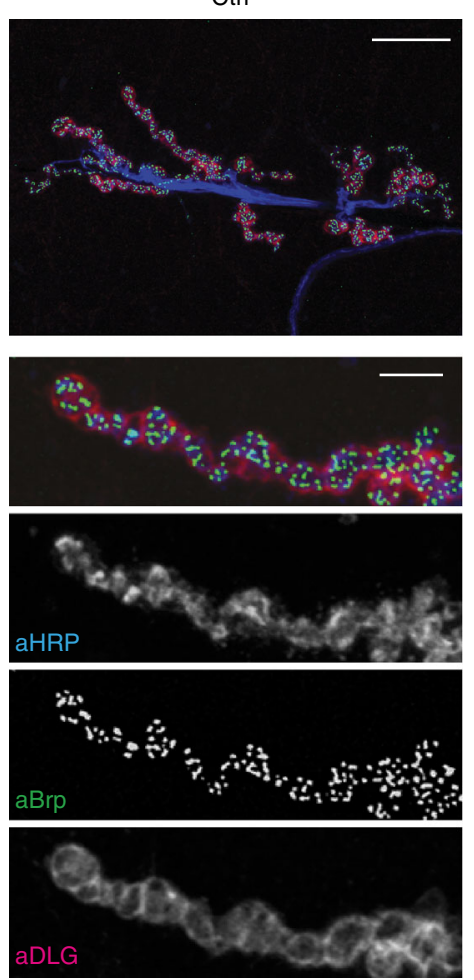

$D T S^{\text {pre }}$
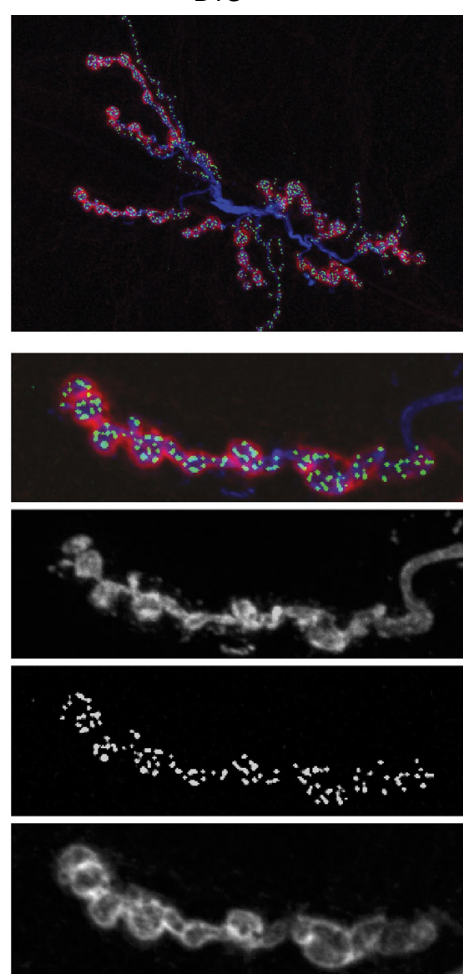

b
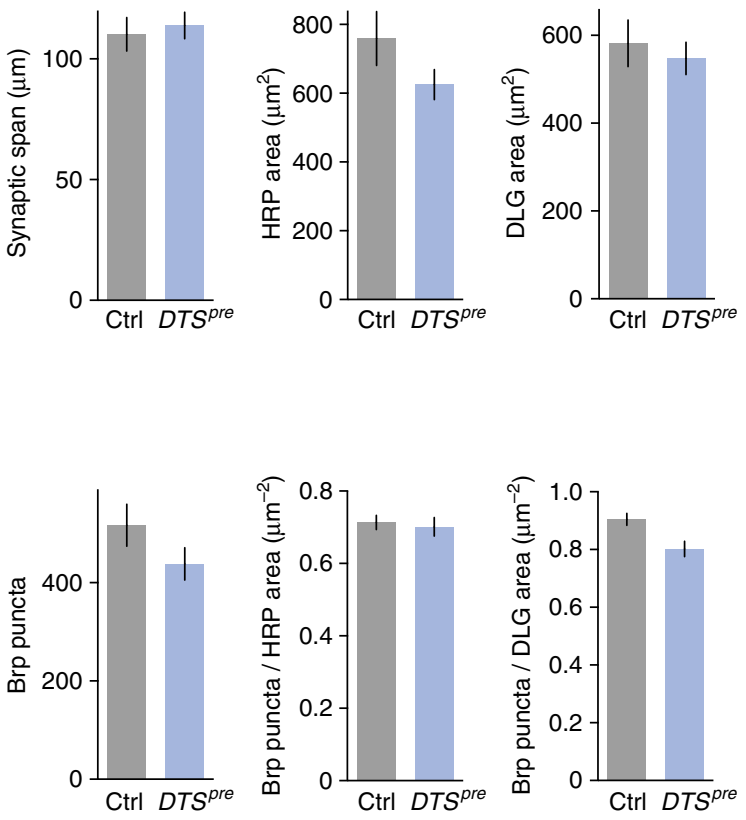

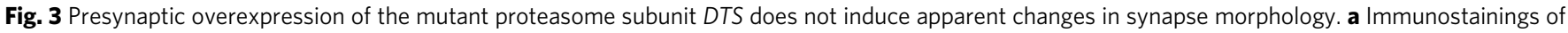
a control NMJ (elav ${ }^{C 155}-$ Gal4) and an NMJ expressing two copies of the mutant proteasome subunit DTS5 presynaptically (elav C155-Gal4; UAS-DTS5; 'DTS ${ }^{\text {prer }}$ ). Larvae were kept at the permissive temperature $\left(25^{\circ} \mathrm{C}\right.$ ) throughout development. NMJs were stained for neuronal membrane (aHRP), postsynaptic reticulum ( $\mathrm{aDLG}$ ) and the active zone marker Bruchpilot (aBrp); scale bar overview $=20 \mu \mathrm{m}$; scale bar inset $=5 \mu \mathrm{m}$. $\mathbf{b}$ Quantification of NMJ parameters for the two genotypes shown in (a). Presynaptic DTS overexpression does not result in apparent changes in synapse morphology. Mean \pm s.e. m.; $n=24$ NMJs; Student's t-test

When comparing EPSCs between DTSpre mutants and controls in the absence of PhTX, we noted that presynaptic DTS overexpression induced an increase in EPSC amplitude as well as a slowing of EPSC decay kinetics (Fig. 4b, c; light gray and light blue data). Together, both effects translate into a significant potentiation of EPSC charge in DTS ${ }^{p r e}$ mutants (Fig. 4b). It is worth noting that changes in EPSC charge are of physiological relevance at this synapse because they translate into changes in EPSP amplitudes due to the relatively slow membrane time constant of the postsynaptic muscle cell $(\tau \approx 40 \mathrm{~ms})^{39}$. The observed increase in EPSC charge upon presynaptic DTS expression could be due to presynaptic and/or postsynaptic changes. If postsynaptic changes underlie the observed phenotype, one would expect corresponding changes in mEPSC amplitude and/or slowing of mEPSC decay kinetics. However, we did not notice corresponding changes in the amplitude, kinetics or charge of mEPSCs in DTS mutants (Fig. 4d, light gray and light blue data). On the contrary, we even observed a significant acceleration of mEPSC decay kinetics in DTS mutants compared to controls (Fig. 4d, middle). These data suggest that presynaptic mechanisms underlie the proteasome perturbationinduced potentiation of EPSC charge.

Proteasome function and PHP modulate EGTA-sensitive vesicles. Presynaptic proteasome interference induced a concomitant increase in EPSC amplitude and a slowing of EPSC decay kinetics, which we attribute to presynaptic changes (Fig. 4). Based on previous work ${ }^{40,41}$, this phenotype may be due to additional release of vesicles that are more 'loosely coupled', i.e., which are physically more distant from $\mathrm{Ca}^{2+}$ channels and/or have a lower intrinsic $\mathrm{Ca}^{2+}$ sensitivity. To test this hypothesis, we probed the effects of the $\mathrm{Ca}^{2+}$ chelator EGTA on release (Fig. $5 \mathrm{a}$, b). Because of its relatively slow $\mathrm{Ca}^{2+}$-binding rate ${ }^{42}$, this buffer predominantly interferes with the exocytosis of 'loosely-coupled' vesicles with a lower $p_{r}$. Application of EGTA-AM (50 $\mu \mathrm{M}$ for 10 min, see Methods) did not significantly change EPSC amplitude or EPSC charge at control synapses, whereas it significantly reduced EPSC charge at $D T S^{p r e}$ mutant synapses (Fig. 5a, b). By contrast, EGTA treatment had no significant effects on mEPSP amplitude (Supplementary Fig. 5a), suggesting that presynaptic proteasome perturbation predominately enhances the release of EGTA-sensitive vesicles.

Based on this result and on the observation that presynaptic proteasome inhibition disrupts PHP (Figs. 1-4), we hypothesized that homeostatic signaling at wild-type synapses may also augment release of EGTA-sensitive vesicles. We therefore next assessed the EGTA sensitivity of release after inducing PHP through PhTX application at wild-type synapses (Fig. 5a, b). Strikingly, EGTA treatment after PhTX application completely blocked the expression of PHP, leading to a pronounced reduction in EPSC amplitude and EPSC charge with respect to synapses that were treated with PhTX alone (Fig. 5a, b). EGTA application had a similar effect on readily releasable vesicle pool (RRP) size assessed by cumulative EPSC amplitude analysis $^{15,43,44}$ (Supplementary Fig. 5e). Whereas PhTX-induced glutamate receptor impairment normally results in an increase in RRP size at control synapses ${ }^{16,44}$ (Supplementary Fig. 5; see below), we observed reduced cumulative EPSC amplitudes at synapses treated with EGTA and PhTX (Supplementary Fig. 5e). 
a Ctrl PhTX DTS pre DTS ${ }^{\text {pre }}+\mathrm{PhTX}$
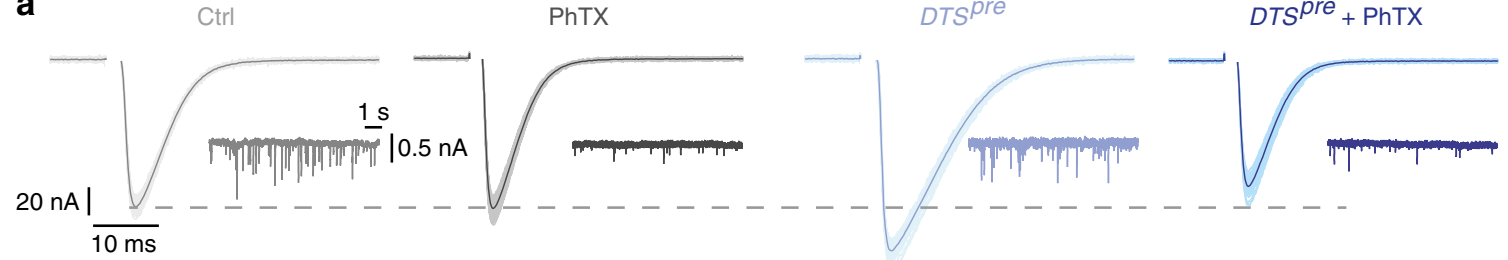

b
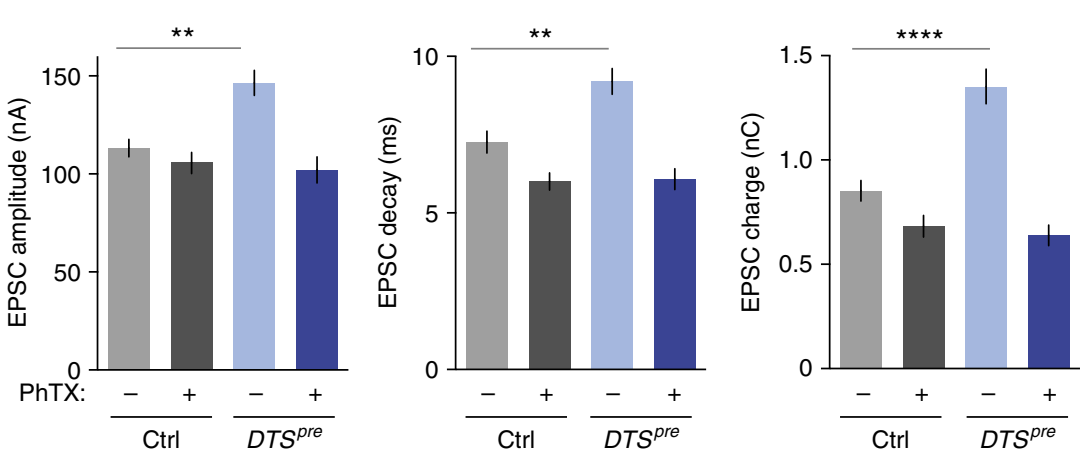

C

d
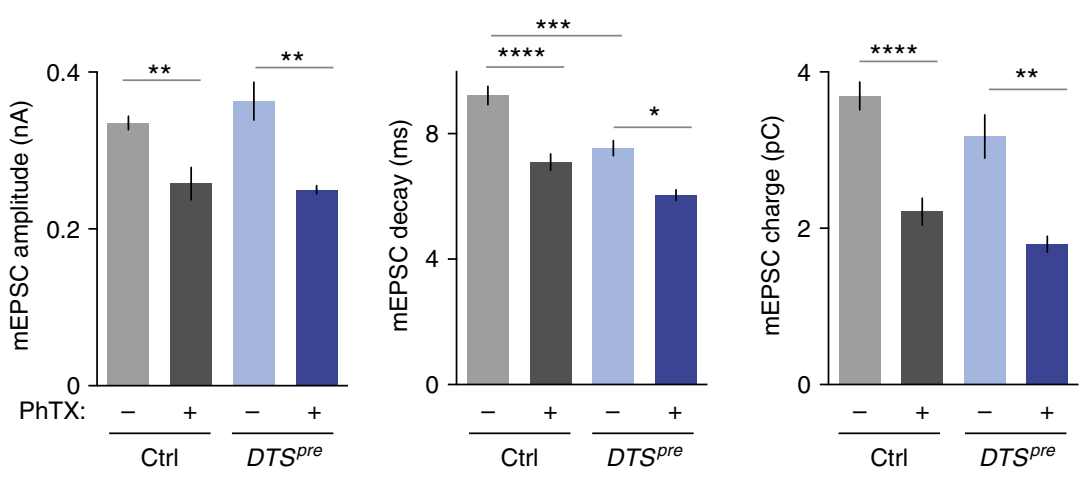

e

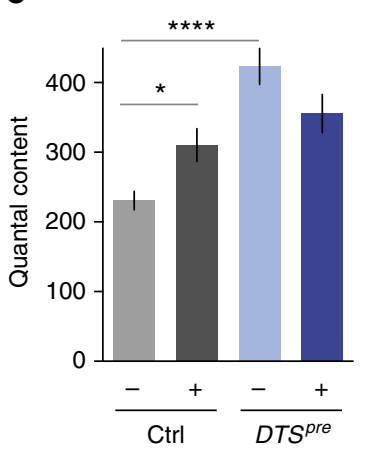

Fig. 4 Presynaptic proteasome perturbation increases EPSC amplitude and decay time. a Two-electrode voltage-clamp recordings of DTSpre mutants and controls with or without PhTX application $\left(20-30 \mu \mathrm{M} ; 1 \mathrm{mM}\left[\mathrm{Ca}^{2+}\right]_{\mathrm{e}}\right.$ ). Representative EPSCs (50 sweeps per cell; the average is displayed in darker color; stimulation artifacts have been blanked for clarity) and mEPSCs are shown. b Quantification of the experiment shown in (a). Note that presynaptic proteasome inhibition leads to an increase in EPSC amplitude, decay time constant and charge. Moreover, proteasome inhibition blocks homeostatic plasticity in response to PhTX application. Mean \pm s.e.m.; $n \geq 10$ cells; ${ }^{\star \star} p<0.001 ;{ }^{\star \star \star *} p<0.00001$; ANOVA and Tukey's multiple comparison tests. c Overlay of an average EPSC of a control cell (gray) and after proteasome inhibition (blue; DTSpre). The dashed line shows the EPSC for DTSpre normalized to the EPSC amplitude of control. Inset: representative mEPSCs for control and DTS pre. d Quantification of mEPSC amplitude, decay and charge. Due to technical reasons, mEPSCs were recorded in different cells than EPSCs. Note that the EPSC decay is faster than the mEPSC decay, likely due to partial glutamate receptor desensitization/saturation. Mean \pm s.e.m.; $n \geq 6$ cells; ${ }^{\star} p<0.05,{ }^{\star \star} p<0.001 ;{ }^{\star \star \star} p<0.0001 ;{ }^{\star \star \star \star} p<0.00001 ;$ ANOVA and Tukey's multiple comparison tests. e Quantal content was calculated by the ratio of EPSC charge/mEPSC charge. Mean \pm s.e.m.; $n \geq 6$ cells; ${ }^{\star} p<0.05$; ${ }^{\star \star \star \star} p<0.00001 ;$ ANOVA and Tukey's multiple comparison tests

Thus, the expression of PHP requires additional release of EGTAsensitive, low- $p_{r}$ vesicles.

We next explored the relationship between EGTA sensitivity of release, PHP and proteasome function. As shown above, control synapses are significantly less EGTA sensitive under baseline conditions than during PHP (Fig. 5a, b). DTS ${ }^{\text {pre }}$-mutant synapses are more EGTA sensitive under baseline conditions than controls (Fig. 5a, b). We next assessed the EGTA sensitivity of release in DTS $^{\text {pre }}$ mutants after PhTX application and found that EGTAAM application led to a decrease in EPSC amplitude and EPSC charge with respect to DTS ${ }^{\text {re }}$ mutants treated with PhTX alone (Fig. 5a, b; two blue bars, right). However, this decrease was significantly less pronounced than the EGTA-induced reduction in EPSC amplitude of PhTX-treated control synapses, indicating that control synapses are more EGTA-sensitive during homeostatic plasticity than DTS ${ }^{p r e}$-mutant synapses. Interestingly, the relative decrease in EPSC amplitude induced by EGTA application after PHP induction was similar between controls and DTS ${ }^{\text {pre }}$ mutants when compared to the respective baseline conditions without PhTX or EGTA treatment (ctrl: $-49 \pm 4 \%$; DTS $^{\text {pre }}:-42 \pm 5 \%$; Fig. 5a: red bars; Fig. $5 \mathrm{~b}$ : compare first and last column of each genotype). This demonstrates that the summed EGTA sensitivity of DTS ${ }^{\text {pre }}$ mutants under baseline conditions and after homeostatic challenge is similar to the EGTA sensitivity of wild-type synapses during PHP. Two conclusions can be drawn. First, these observations are consistent with the idea that proteasome function and PHP converge on EGTA-sensitive vesicles. Second, our data suggest that homeostatic signaling regulates EGTA-sensitive vesicles more potently than proteasome function.

Proteasome perturbation increases $\mathrm{Ca}^{2+}$ influx and RRP size. It has been shown that PHP requires an increase in presynaptic 
a

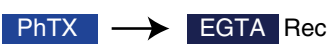

Ctrl

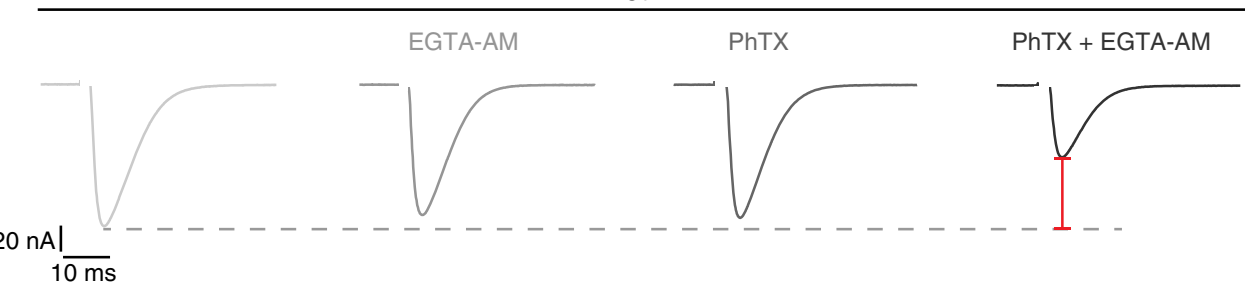

$\overline{10 \mathrm{~ms}}$

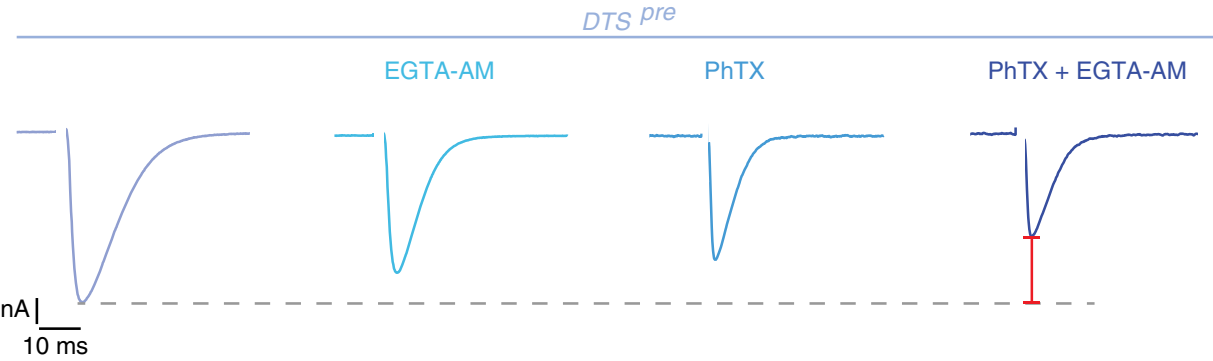

b
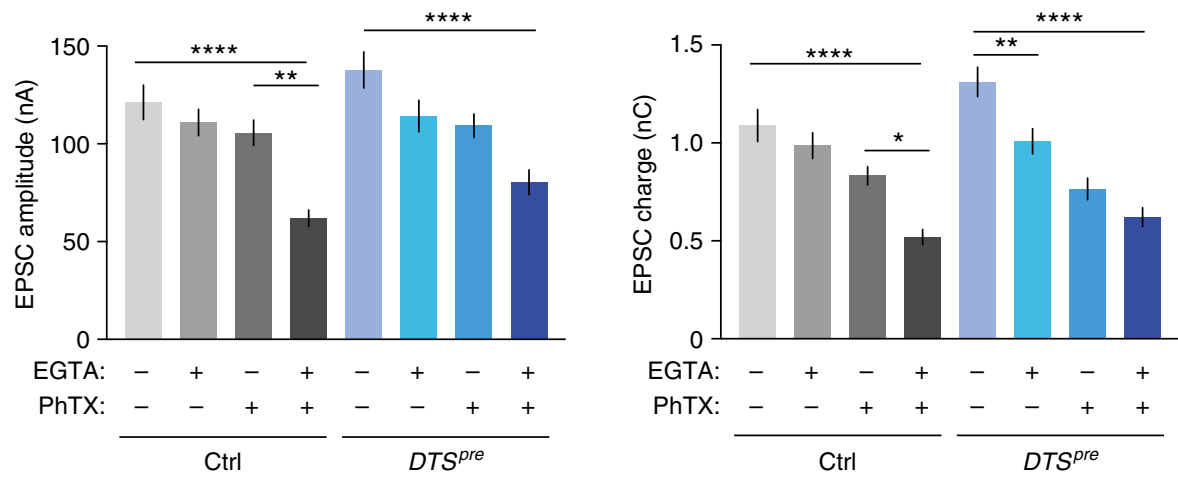

Fig. 5 Glutamate receptor perturbation and proteasome perturbation enhance release of EGTA-sensitive vesicles. a Representative EPSC traces of control (elav ${ }^{C 155}$,'ctrl') and DTS pre (elav ${ }^{\mathrm{C} 155}$-Gal4; UAS-DTS5; 'DTSpre') synapses. The two left EPSC traces from each genotype were recorded from synapses treated with $50 \mu \mathrm{M}$ EGTA-AM for 10 min or DMSO as control. The last two EPSC traces were recorded from synapses treated with $30 \mu \mathrm{M}$ PhTX for 10 min, followed by wash and treatment with $50 \mu \mathrm{M}$ EGTA-AM or DMSO for $10 \mathrm{~min}\left(1 \mathrm{mM}\left[\mathrm{Ca}^{2+}\right]_{\mathrm{e}}\right)$. The red bars symbolize that the decrease after PhTX and EGTA application compared to untreated cells is similar in both genotypes. $\mathbf{b}$ Quantification of EPSC amplitude and EPSC charge of the experiment shown in (a). EGTA-AM application induces a significant decrease in EPSC charge in DTS pre mutants, whereas there is no significant decrease in EPSC charge at control synapses. After PhTX and EGTA treatment, EPSC amplitudes are significantly smaller in controls than in DTSpre mutants ( $p=0.0197$; unpaired $t$ test). By contrast, there is no significant difference between both groups in the absence of EGTA treatment $(p=0.7)$, indicating that controls are more EGTA sensitive during homeostatic plasticity than DTS pre mutants. The increased EGTA-AM sensitivity after PhTX incubation implies that the induction of PHP requires increased recruitment of EGTA-sensitive, low- $p_{r}$ vesicles. Mean \pm s.e.m.; $n \geq 10$ cells; ${ }^{*} p<0.05 ;{ }^{* \star} p<0.001 ;{ }^{* \star *} p<0.00001 ;$ ANOVA and Tukey's multiple comparison tests

$\mathrm{Ca}^{2+}$ influx ${ }^{45}$ and RRP size ${ }^{16,44}$. We therefore investigated if release potentiation upon proteasome perturbation involves similar mechanisms. First, we recorded spatially averaged $\mathrm{Ca}^{2+}$ transients at presynaptic boutons in response to single-AP stimulation using two-photon imaging (Fig. 6a-d; terminals were co-loaded with OGB-1 and Alexa-568; see Methods). As shown in Fig. 6b, c, DTS pre synapses had significantly increased peak amplitudes of presynaptic $\mathrm{Ca}^{2+}$ transients with respect to controls. The magnitude of the increase in presynaptic $\mathrm{Ca}^{2+}$ influx after proteasome inhibition is relatively large as compared to the effect on neurotransmitter release (Fig. 4a). This observation is in agreement with our electrophysiology data showing that proteasome impairment predominantly potentiates release of EGTAsensitive, low- $p_{r}$ vesicles in addition to high- $p_{r}$ vesicles (Fig. 5). In DTS ${ }^{p r e}$ mutants, we also detected a significant increase in baseline OGB-1-fluorescence intensity before stimulus onset without corresponding changes in Alexa-568-fluorescence intensity (Fig. 6d, left), suggesting that presynaptic proteasome impairment elevates baseline $\mathrm{Ca}^{2+}$ levels. $\mathrm{Ca}^{2+}$-transient decay kinetics were similar between mutant and control synapses, indicating that DTS expression does not alter $\mathrm{Ca}^{2+}$ extrusion and/or $\mathrm{Ca}^{2+}$ buffering (Fig. 6d, right). Together, these data demonstrate that presynaptic proteasome perturbation results in increased presynaptic $\mathrm{Ca}^{2+}$ influx upon AP stimulation, as well as elevated $\mathrm{Ca}^{2+}$ levels at rest. This indicates that presynaptic proteasome function negatively regulates presynaptic $\mathrm{Ca}^{2+}$ signaling under baseline conditions.

Next, we investigated RRP size using cumulative EPSC analysis during brief $(1 \mathrm{~s}) 60 \mathrm{~Hz}$ trains ${ }^{15,43,44}$. Due to the observation that $D S^{p r e}$ expression potentiates EPSC charge (Fig. 4), we based our RRP estimate on cumulative EPSC charge. As shown in Fig. 6e, f, $\mathrm{RRP}$ analysis of DTS ${ }^{\text {re }}$ mutants revealed a significant increase in 
apparent RRP size with respect to controls. By contrast, there was no significant change in short-term depression during $60-\mathrm{Hz}$ stimulation between DTS ${ }^{\text {re }}$ mutants and controls (Supplementary Fig. 5b), suggesting that the increase in RRP size is unlikely to be mainly due to enhanced presynaptic $\mathrm{Ca}^{2+}$ influx. The conclusion that DTS pre also affects release downstream of presynaptic $\mathrm{Ca}^{2+}$ influx is further supported by the observation that an increase in $\left[\mathrm{Ca}^{2+}\right]_{\mathrm{e}}$ from $1 \mathrm{mM}$ to $2 \mathrm{mM}$ does not result in a slowing of EPSC decay kinetics at wild-type synapses (Supplementary Fig. 5c), in contrast to DTS ${ }^{\text {pre }}$ mutants (Fig. 4b). We conclude that the presynaptic proteasome opposes release of low- $p_{r}$ vesicles through similar mechanisms employed during PHP: the modulation of presynaptic $\mathrm{Ca}^{2+}$ influx and RRP size.

Our data imply that presynaptic proteasome impairment predominately recruits vesicles with a low $p_{r}$ (Fig. 4) that are EGTA sensitive (Fig. 5). To provide further support for this a

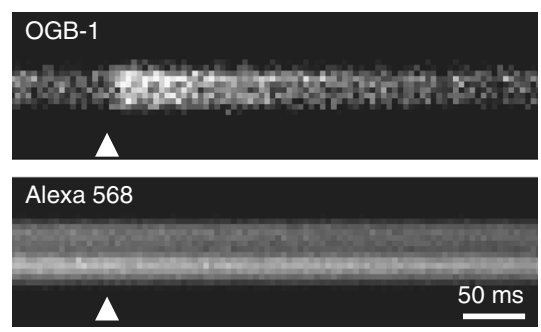

C
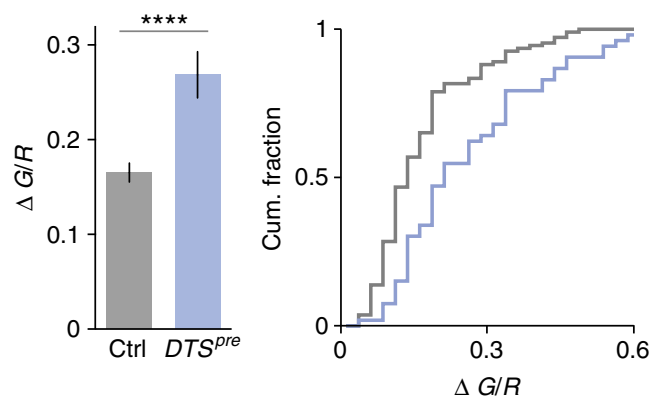

e
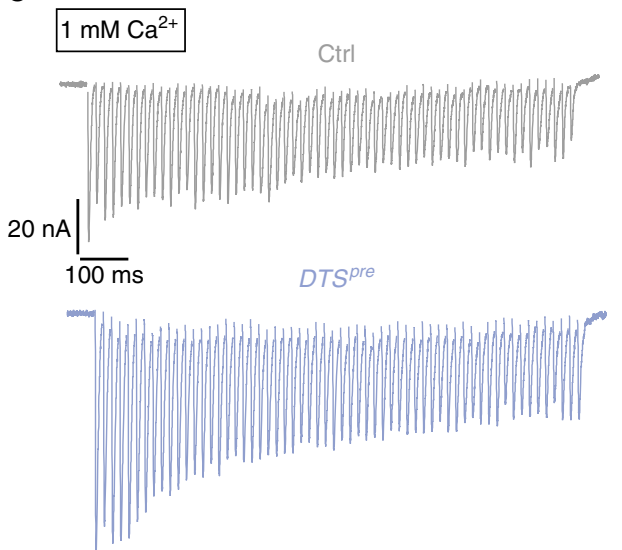

g

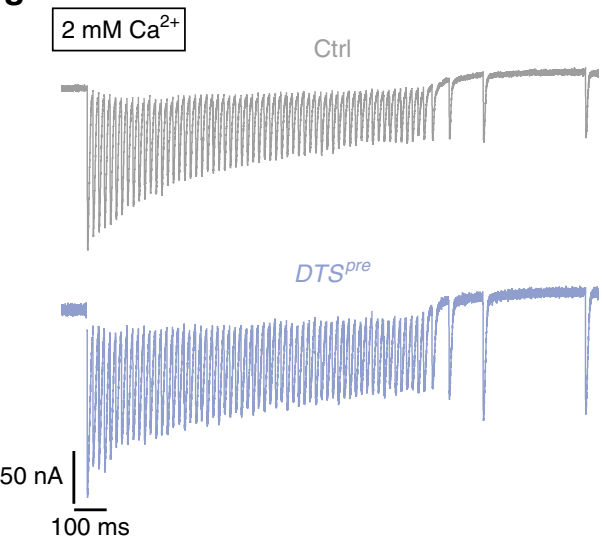

b

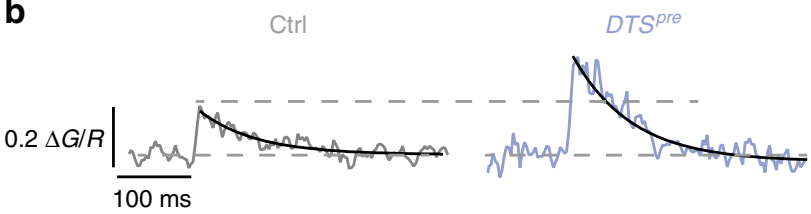

d
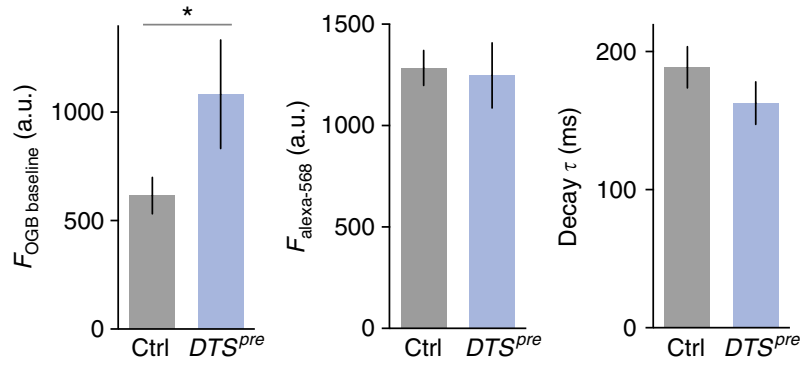
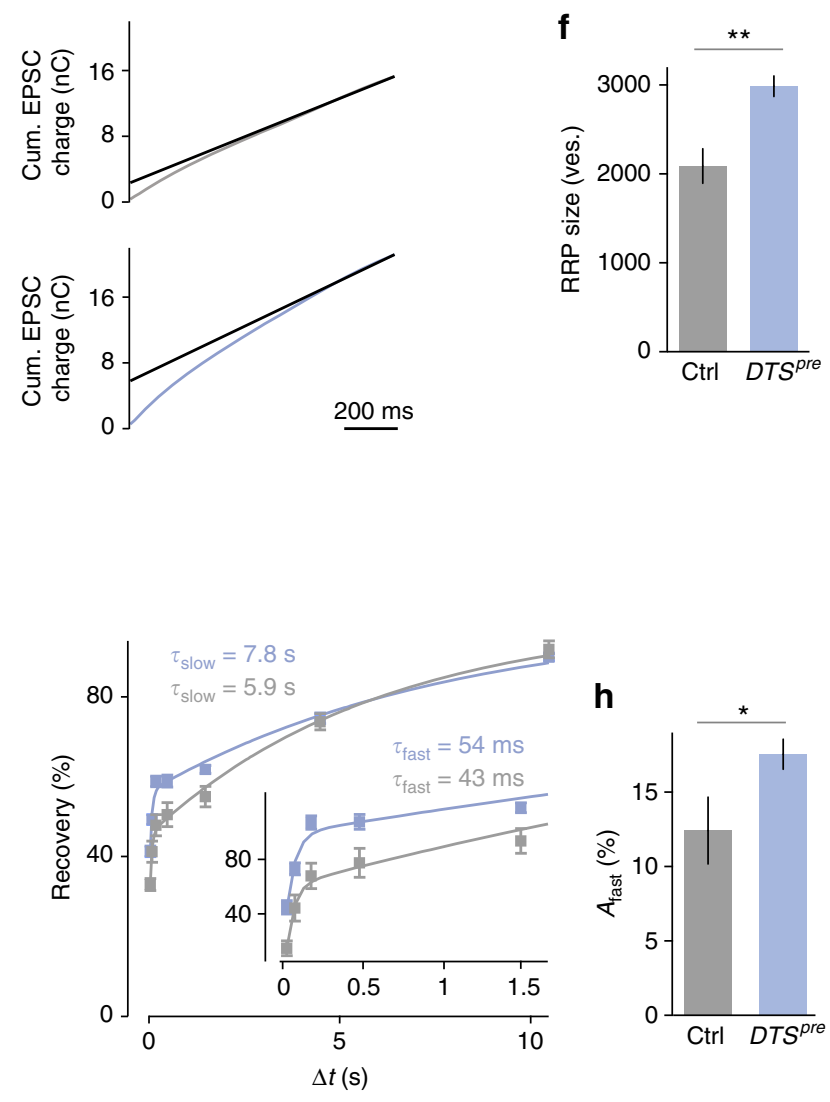

h

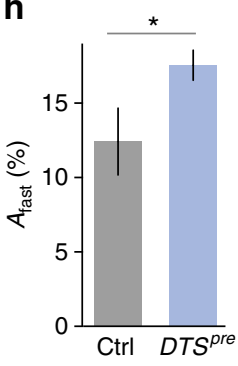


model, we assayed vesicle resupply after RRP depletion induced by high-frequency stimulation at elevated $\mathrm{Ca}^{2+}$ concentrations $\left(2 \mathrm{mM}\left[\mathrm{Ca}^{>2+}\right]_{e} ;\right.$ Fig. $\left.6 \mathrm{~g}\right)$. At many synapses, there are two distinct kinetic components of vesicle replenishment following high-frequency stimulation: a fast recovery time component that likely corresponds to resupply of vesicles with a relatively low $p_{r}$, and a slow recovery period that is thought to reflect replenishment of vesicles with a higher $p_{r}{ }^{46-48}$. We therefore hypothesized that proteasome perturbation may predominately enhance the fast recovery component. DTS ${ }^{p r e}$ mutants displayed a significantly increased amplitude of the fast recovery component as compared to controls (Fig. 6g, h). There were no significant changes in the fast and slow recovery time constants, as well as the amplitude of the slow recovery component between DTS ${ }^{\text {pre }}$ mutants and controls. These data provide indirect evidence that proteasome inhibition results in increased release of low- $p_{r}$ vesicles that recover rapidly.

dysbindin and rim are required for release potentiation. Proteasome perturbation blocks PHP and enhances $\mathrm{Ca}^{2+}$ influx and neurotransmitter output. To genetically link proteasome function-dependent control of release and homeostatic plasticity, we assayed synaptic transmission before and after lactacystin application in several PHP mutants (Fig. ${ }^{49}$ ). For quantification, we normalized the mean EPSP amplitude after lactacystin incubation $(100 \mu \mathrm{M}$ for $15 \mathrm{~min})$ to the EPSP amplitude after incubation with saline for $15 \mathrm{~min}$ (for absolute data see Table S1). As shown in Fig. 7a, b, most PHP mutants displayed an increase in EPSP amplitude after proteasome interference that was similar to wild-type control synapses (black/gray data). Interestingly, two mutants, rab-3-interacting-protein (rim) and dysbindin (dysb), did not show any change in either EPSP amplitude or quantal content after lactacystin application (red data; Fig. 7a-c). This indicates that these two homeostatic plasticity genes are required for the increase in release upon proteasome inhibition, and therefore provide a link between homeostatic and proteasome-dependent control of release. By extension, based on our observation that proteasome perturbation failed to enhance release in specific PHP mutants, we suggest that the presynaptic proteasome regulates specific presynaptic proteins that are required for homeostatic plasticity.

Genetic interaction between dysbindin and DTS during PHP. In a next set of experiments we investigated the role of dysbindin in proteasome-dependent control of release in more detail (Figs. $7 \mathrm{~d}$, e, and 8). We focused on dysbindin because dysbindin mutants do not have major defects in baseline synaptic transmission $^{11}$, and because the human dysbindin homolog (DTNBP1) has been linked to schizophrenia ${ }^{50}$. First, we aimed at providing a genetic link between dysbindin and proteasome function in the context of homeostatic plasticity. PHP is completely blocked in homozygous dysbindin mutants ${ }^{1}$ (Fig. 8h, i) or after presynaptic expression of two DTS5 copies (Fig. 4). Thus, analysis of homozygous double mutants would not be informative. We therefore tested PhTX-induced PHP in heterozygous double mutants lacking one dysbindin copy and expressing one DTS copy presynaptically (elav ${ }^{c 155}$-Gal-4/Y;DTS5/+,dysb/+; Fig. 7d, e). A prerequisite for this analysis is that the heterozygous mutants alone do not impair PHP. PHP proceeds normally in heterozygous dybindin mutants ${ }^{11}$. Similarly, PhTX application induced a homeostatic increase in quantal content that restored EPSP amplitudes to baseline levels at synapses expressing one DTS5 copy (Fig. $7 \mathrm{~d}, \mathrm{e}$ ). However, when examining DTS5/+,dysb/+ double heterozygous animals, we observed significantly smaller EPSP amplitudes and no significant increase in quantal content after PhTX incubation (Fig. 7d, e). This finding demonstrates a genetic interaction between dysbindin and DTS during homeostatic plasticity. We also noted a significant increase in EPSP amplitude in DTS5/+,dysb/+ double heterozygous mutants compared to DTS5/+ heterozygous mutants in the absence of PhTX. This suggests that mild proteasome impairment can potentiate release at synapses with decreased dysbindin expression under baseline conditions, but not during homeostatic plasticity. Together, our results demonstrate a genetic link between dysbindin and proteasome function during PHP and baseline synaptic transmission.

Dysbindin controls release of EGTA-sensitive vesicles. Acute proteasome perturbation failed to potentiate release at homozygous dysbindin mutant synapses (Fig. 7). We therefore hypothesized that Dysbindin protein levels may be important for the regulation of neurotransmitter release. To test this hypothesis, we first assayed release after presynaptic overexpression of wild-type venus-dysbindin (Fig. 8a-g). A similar construct was previously shown to rescue the dysbindin mutant phenotype and to potentiate release ${ }^{11}$. Consistent with previous data ${ }^{11}$, venustagged Dysbindin localized to presynaptic NMJ boutons (Fig. 8a). We detected a significant increase in EPSC amplitude and EPSC charge upon single-AP stimulation (Fig. 8b, c), as well as a significant increase in cumulative EPSC amplitude during $60 \mathrm{~Hz}$ trains upon presynaptic dysbindin overexpression (Fig. 8d, e), indicating a role for $d y s b i n d i n$ expression levels in the regulation of RRP size.

We provided evidence that both genetic proteasome perturbation and PHP induction result in increased recruitment of EGTAsensitive vesicles (Fig. 5). We next asked if the increase in release seen after dysbindin overexpression is also due to enhanced

Fig. 6 Proteasome perturbation increases presynaptic $\mathrm{Ca}^{2+}$ influx, RRP size and the amplitude of the fast recovery phase. a Representative two-photon line scans of a control bouton after loading with OGB-1 and Alexa-568. Arrowhead indicates AP-stimulus onset. b Representative spatially averaged Ca ${ }^{2+}$ transients of a control synapse and a DTS pre synapse (average of 10 sweeps). c Quantification of $\mathrm{Ca}^{2+}{ }^{2}$ transient peak amplitudes of control and DTS pre synapses and cumulative frequency plot. Proteasome inhibition results in a significant increase in the peak amplitude compared to control cells. $\mathbf{d}$ Baseline fluorescence of the $\mathrm{Ca}^{2+}$ indicator OGB-1 and the $\mathrm{Ca}^{2+}$-independent dye Alexa-568 in the two genotypes. Baseline OGB-1 fluorescence is significantly higher in DTS pre synapses. The decay time constant $\tau$ is not changed. Mean \pm s.e.m.; $n \geq 53$ boutons; ${ }^{\star} p<0.05 ;{ }^{\star \star \star \star} p<0.00001$; Wilcoxon rank-sum test. e Left: Representative EPSC trains in response to $60-\mathrm{Hz}$ stimulation ( 60 stimuli, $1 \mathrm{mM}\left[\mathrm{Ca}^{2+}\right]_{\mathrm{e}}$ ) for control cells and DTSpre mutant cells. Right: cumulative EPSC charge was calculated by back-extrapolation of a linear fit (black line) to the last 15 stimuli of the cumulative EPSC integrals to time point zero. $\mathbf{f}$ RRP size was calculated by dividing cumulative EPSC charge by the average mEPSC charge. $\mathbf{g}$ Left: Example of EPSCs during $60-\mathrm{Hz}$ stimulation (60 stimuli, 2 $\mathrm{mM}\left[\mathrm{Ca}^{2+}\right]_{\mathrm{e}}$ ) followed by recovery pulses given at intervals of $25,75,175$ and $475 \mathrm{~ms}$ after the last train stimulus of the indicated genotypes. Right: Average recovery (mean EPSC amplitude at a given interval divided by the first EPSC amplitude of the $60 \mathrm{~Hz}$ train) versus recovery interval $(\Delta t)$ of the indicated genotypes superimposed with biexponential fits. Inset shows the data at short intervals. Biexponential fits of average data gave the time constants noted in the figure. $\mathbf{h}$ Average amplitude of the fast recovery component ' $A_{\text {fast }}$ ' of the two groups obtained from bi-exponential fits of individual synapses. Note the significant increase in the fast recovery component in DTS pre mutants (there was no difference in $A_{\text {slow }}$ and recovery kinetics between the two groups). Mean \pm s.e.m.; $n \geq 10$ cells; ${ }^{\star} p<0.05 ;{ }^{\star \star} p<0.001$; Student's $t$-test 
a

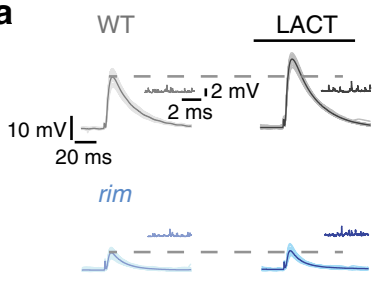

$d y s b$

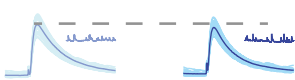

b

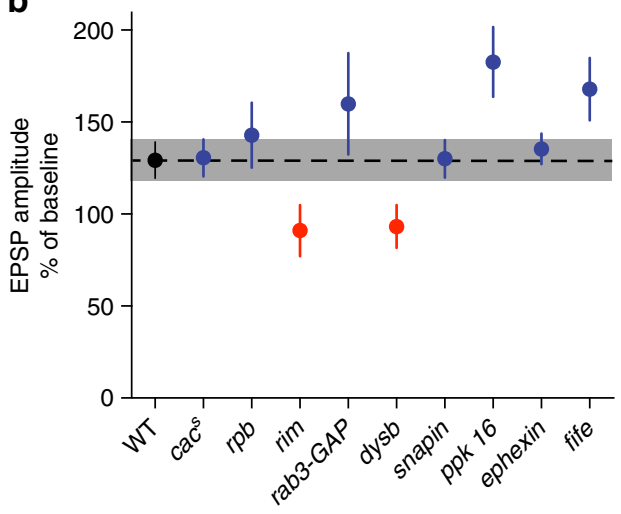

C
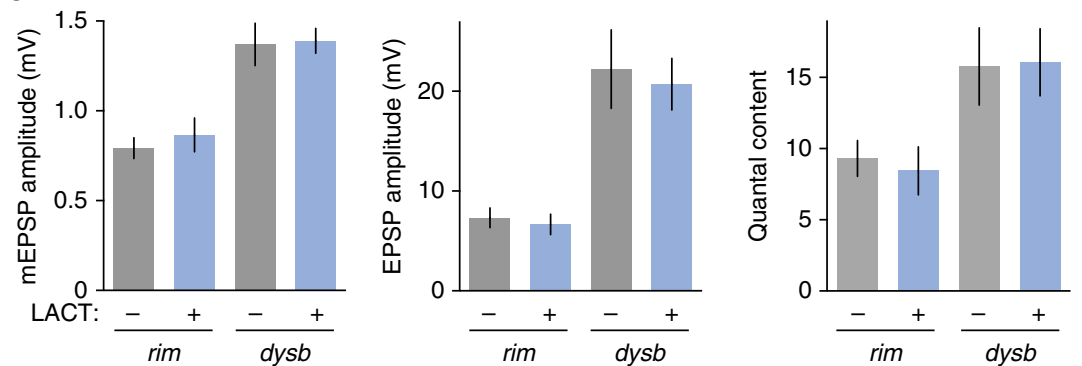

d

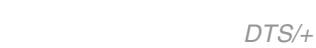

DTS $/+$

$+\mathrm{PhTX}$

DTS/dysb $+\mathrm{PhTX}$

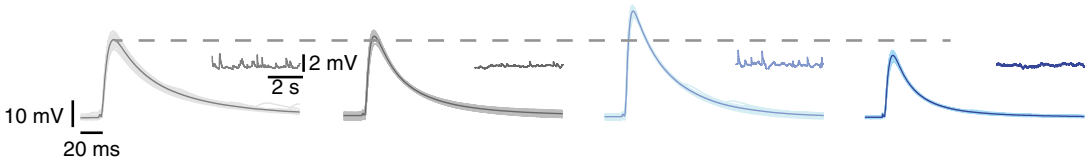

e
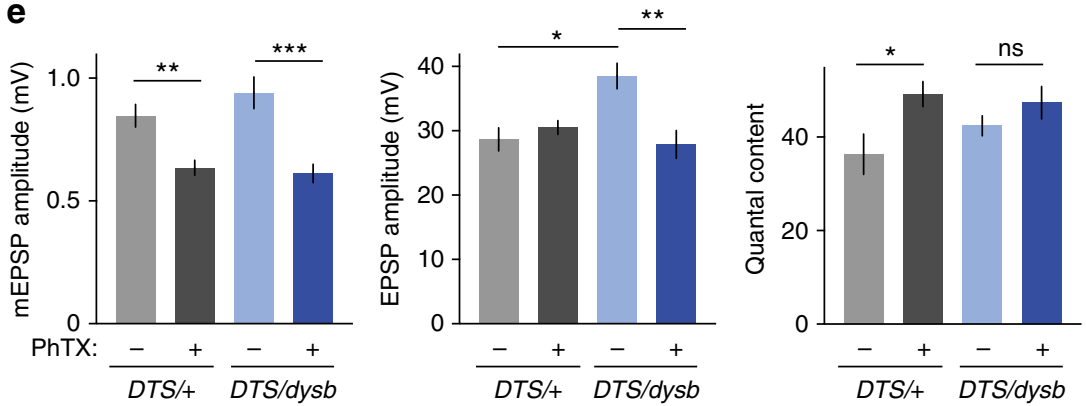

Fig. $7 \mathrm{rim}$ and dysbindin are required for proteasome-dependent potentiation. a Application of $100 \mu \mathrm{M}$ lactacystin for 15 min to wild-type synapses and selected PHP mutants. Representative EPSP and mEPSP traces are shown for wild type, rim and dysbindin mutants. b For quantification, EPSP amplitude after lactacystin application was normalized to the EPSP amplitude at baseline. Wild-type EPSPs show an increase to 130\% upon lactacystin treatment. The PHP mutants $c a c^{s}$, rim-binding protein ( $\left.r b p\right)$, rab3-GAP, snapin (presynaptic RNAi), pickpocket16 (ppk 16), ephexin and fife show similar or stronger increases, whereas rim and dysbindin (dysb) mutants do not respond to lactacystin treatment. Due to defects in baseline EPSP amplitude in some of the genotypes, different $\left[\mathrm{Ca}^{2+}\right]_{\mathrm{e}}$ concentrations were used to adjust baseline EPSP amplitudes to comparable values: WT: $0.3 \mathrm{mM}$; cac: $0.5 \mathrm{mM}$; rbp: $1 \mathrm{mM}$; rim: $0.3 \mathrm{mM}$; rab3-GAP: $0.3 \mathrm{mM}$; dysbindin: $0.3 \mathrm{mM}$; snapin $R N A_{i}$ : $0.3 \mathrm{mM}$; pickpocket 16: $0.25 \mathrm{mM}$; ephexin: $0.25 \mathrm{mM}$; fife: $0.3 \mathrm{mM}$. Mean \pm s.e.m.; $n \geq 8$ cells. c Quantification of mEPSP amplitude, EPSP amplitude and quantal content of rim and dysbindin mutant synapses at baseline and after lactacystin application (100 $\mu \mathrm{M}$ for $15 \mathrm{~min})$. Mean \pm s.e.m.; $n \geq 10$ cells. $\mathbf{d}$ Synapses expressing one allele of the mutant proteasome subunit presynaptically either in a wild-type background (DTS/+; het) or in larvae mutant for one allele of dysbindin (DTS/dysb; double het) were measured under baseline conditions and after application of $20 \mu \mathrm{M}$ PhTX for $10 \mathrm{~min}\left(0.3 \mathrm{mM}\left[\mathrm{Ca}^{2+}\right]_{\mathrm{e}}\right)$. Representative EPSP and mEPSP traces are shown. e Quantification of mEPSP amplitude, EPSP amplitude and quantal content for the experiment described in (d). DTS/+heterozygous synapses have no defect in PHP, whereas DTS/dysb double heterozygous synapses show no increase in quantal content after PhTX application. ${ }^{\star} p<0.05,{ }^{\star \star} p<0.001 ;{ }^{\star \star \star} p<0.0001$

recruitment of EGTA-sensitive vesicles. Application of EGTA$\mathrm{AM}$ to synapses overexpressing venus-dysbindin resulted in a significant decrease in EPSC amplitude (Fig. 8f, g), similar to the decrease seen after EGTA-AM application to DTS pre synapses (Fig. 5). EGTA-AM treatment of dysbindin-overexpressing synapses also significantly reduced cumulative EPSC amplitudes compared to untreated NMJs overexpressing dysbindin (Supplementary Fig. 5e). These results suggest that $d y$ sbindin overexpression potentiates release from an EGTA-sensitive vesicle pool. 
Wild-type synapses display a high EGTA sensitivity upon PhTX treatment (Fig. 5). If dysbindin is involved in homeostatic regulation of EGTA-sensitive vesicles, we expect release to be less EGTA sensitive after PhTX application in dysbindin mutants. To test this hypothesis, we recorded EPSCs in dysbindin mutants after incubation with PhTX and PhTX+EGTA-AM (Fig. 8h, i).
First, we observed that baseline synaptic transmission was not changed in dysbindin mutants compared to wild-type controls (Fig. $8 \mathrm{~h}$, i, left; $1 \mathrm{mM}\left[\mathrm{Ca}^{2+}\right]_{\mathrm{e}}$ ), in line with a previous study ${ }^{11}$. Application of PhTX led to a significant decrease in EPSC amplitude and EPSC charge, indicating that PHP is impaired in dysbindin mutants, consistent with earlier work ${ }^{11}$. However, and

a
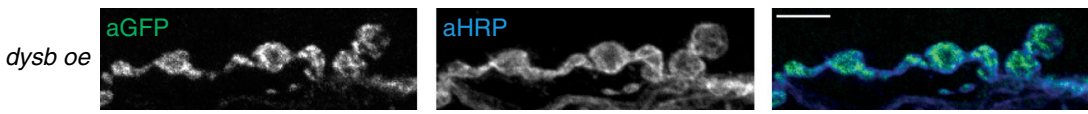

b
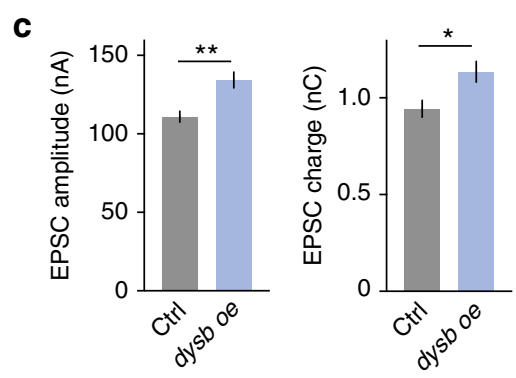

d
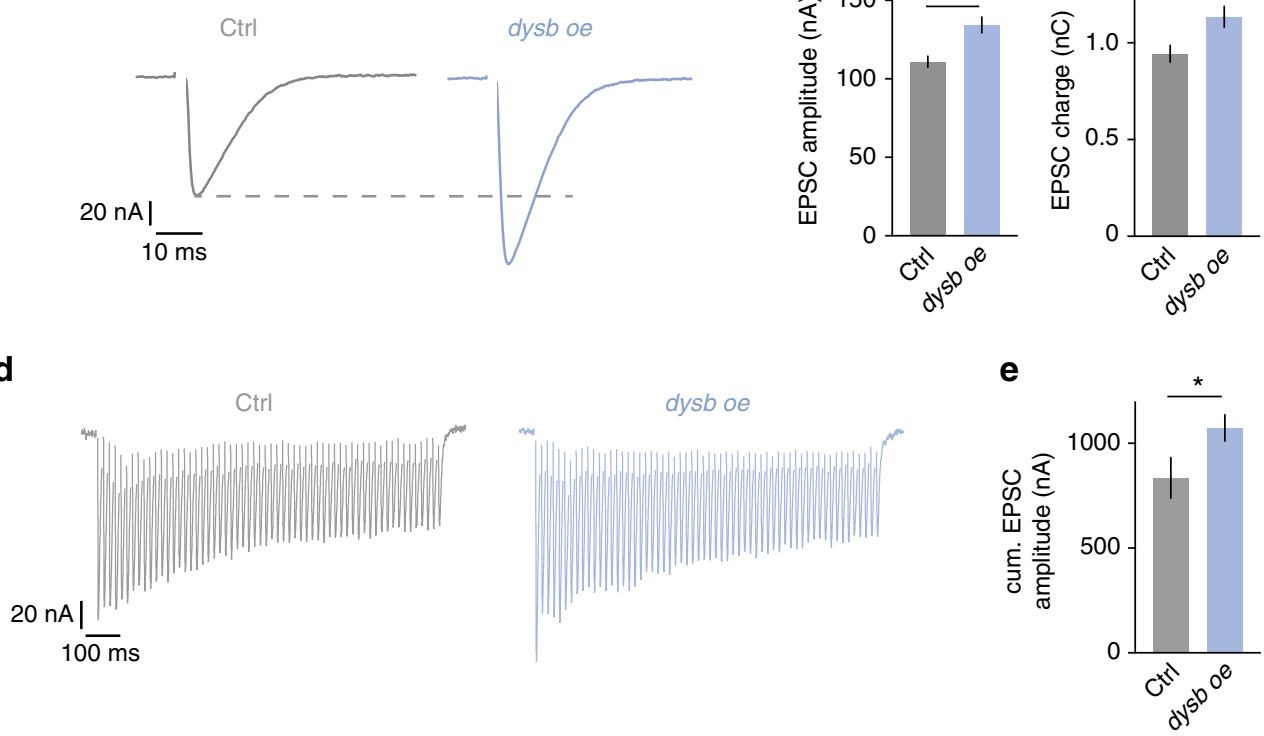

$\mathbf{f}$
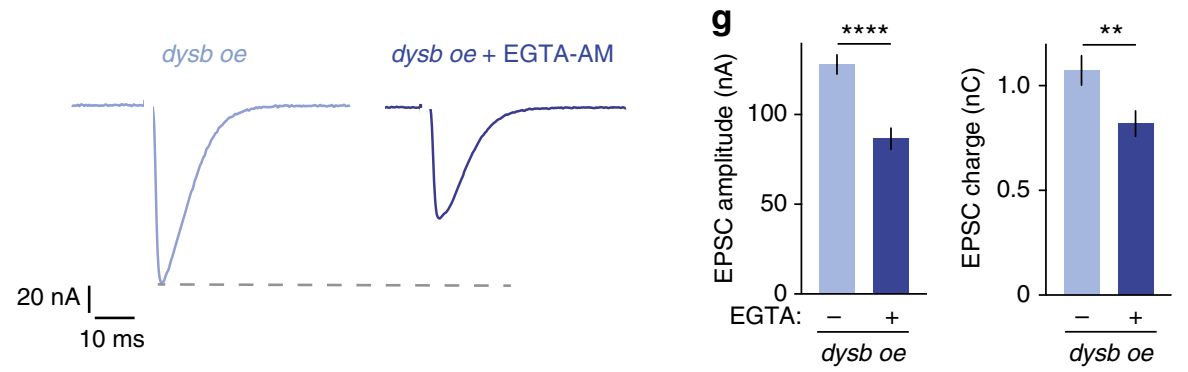

h
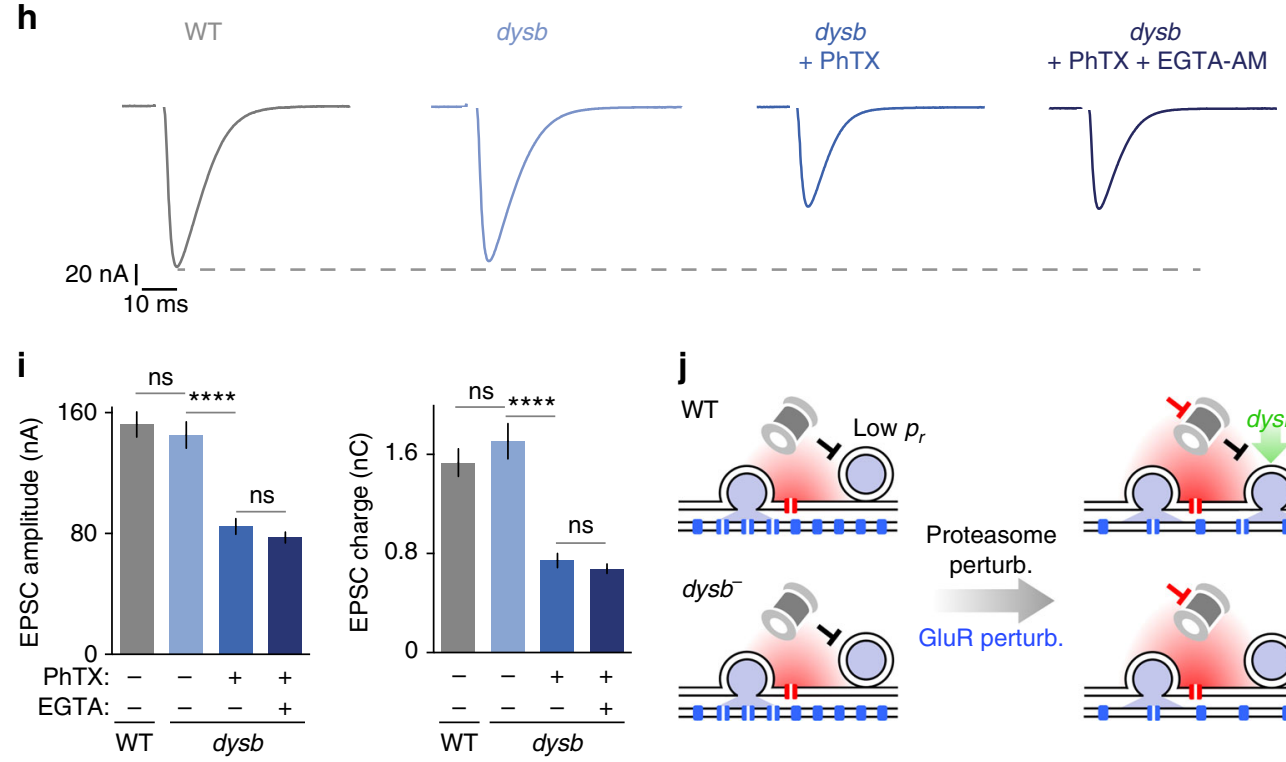

j
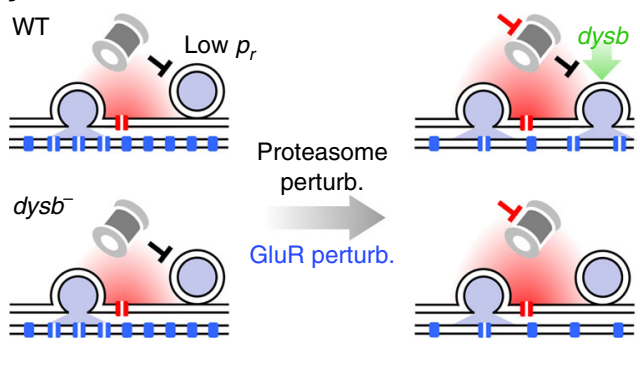
in contrast to wild-type synapses, application of EGTA-AM onto PhTX-treated dysbindin mutants did not result in significant changes in EPSC amplitude or charge (Fig. 8h, i). These data support the conclusion that the enhanced release of EGTAsensitive vesicles during PHP requires $d y$ sbindin.

\section{Discussion}

Here we demonstrate that presynaptic proteasome function is indispensable for PHP induced by glutamate receptor perturbation at the Drosophila NMJ. Moreover, we provide evidence that an EGTA-sensitive pool of synaptic vesicles is regulated by proteasome function, homeostatic plasticity and dysbindin.

We observed rapid effects of proteasome perturbation on neurotransmitter release and the abundance of ubiquitinated proteins on the minute time scale. These data suggest that the proteasomal degradation rate is relatively high under baseline conditions, consistent with previous work on local protein degradation in the presynaptic or postsynaptic compartment ${ }^{2,3}$, and considerably faster than the average neuron-wide turnover rates of synaptic proteins $\left(2-5\right.$ days $\left.{ }^{1}\right)$. Such rapid degradation rates could in turn allow for potent modulation of protein abundance and/or ubiquitination through regulation of UPS function during PHP.

Perturbation of proteasome function has diverse effects on cellular physiology, such as altering the levels of free ubiquitin and mono-ubiquitinated proteins, activating macroautophagy or upregulation of lysosomal enzyme levels ${ }^{1-53}$. The observed phenotypes could thus be due to indirect effects of impaired proteasome function on synaptic physiology. Even if we cannot rule out this possibility, several lines of evidence argue against a major contribution of indirect effects. First, acute or prolonged proteasome perturbation does not affect release at synapses that express PHP (Fig. 2). Second, we provide genetic evidence that proteasome perturbation-induced changes in release are blocked in two PHP mutants (Fig. 7). Third, we detect no major changes in synaptic morphology or synaptic development upon prolonged proteasome perturbation (Fig. 3). Fourth, interfering with proteasome function does not impair neurotransmitter release, but rather results in a net increase in release (Figs. 1, 2, 4, 5, 6 and 7). Taken together, these data suggest that proteasome function opposes release by degrading proteins under baseline conditions, and that normal degradation of these proteins is required for PHP.

Our genetic data imply that not all proteins required for PHP are regulated through UPS-dependent degradation under our experimental conditions (pharmacological proteasome perturbation for $15 \mathrm{~min}$ ), because release can be potentiated upon brief proteasome inhibition in most PHP mutants (Fig. 7). This observation is consistent with a recent study demonstrating that the abundance of most synaptic proteins does not change after prolonged pharmacological proteasome perturbation in cultured mouse hippocampal neurons ${ }^{52}$. Based on our observation that PHP is blocked in cut-up mutants (Supplementary Fig. 2), which were recently shown to have a defect in proteasome trafficking ${ }^{36}$, it is conceivable that proteasome mobility and/or recruitment are modulated during PHP.

We provide evidence that proteasome perturbation and homeostatic signaling recruit EGTA-sensitive vesicles with a lower $p_{r}$ in addition to vesicles with higher $p_{r}$. Previous work revealed that tightly-coupled, high- $p_{r}$ vesicles are required for $\mathrm{PHP}^{16}$. PHP therefore likely involves vesicle pools with different $p_{r}$. Homeostatic regulation of EGTA-sensitive, loosely-coupled vesicles depends on dysbindin (this study), whereas tightlycoupled vesicles are controlled by RIM-binding protein ${ }^{16}$. Together, these results imply that PHP involves two genetically separable populations of vesicles with different $p_{r}$.

Vesicle pools with different $p_{r}$ and release kinetics have been observed at various synapses ${ }^{40,46-48,54}$, and these pools might be differentially regulated during synaptic plasticity. Here we provide evidence that dysbindin is required for the recruitment of EGTA-sensitive vesicles during PHP. It will be exciting to investigate the roles of other presynaptic proteins that have been implicated in the release of EGTA-sensitive vesicles, such as Tomosyn ${ }^{54}$, in the context of proteasome degradation and PHP. Interestingly, a recent study at the mouse NMJ observed accelerated release kinetics of 'slow' synaptic vesicles during $\mathrm{PHP}^{55}$ Thus, homeostatic potentiation of vesicles with lower $p_{r} /$ slower release kinetics may be an evolutionarily conserved mechanism.

Which mechanisms could potentiate the release of low- $p_{r}$ vesicles? We uncovered that presynaptic proteasome perturbation results in enhanced presynaptic $\mathrm{Ca}^{2+}$ influx, independent of major changes in $\mathrm{Ca}^{2+}$ buffering and/or extrusion. We therefore consider changes in presynaptic $\mathrm{Ca}^{2+}$ influx as a possible mechanism underlying the increase in low- $p_{r}$ vesicle release. Proteasome perturbation also increased RRP size. Earlier work revealed that changes in presynaptic $\mathrm{Ca}^{2+}$ influx modulate release in part by altering apparent RRP size ${ }^{16,43,56}$. The increase in RRP size or EGTA sensitivity upon proteasome inhibition may therefore be in part a secondary consequence of enhanced presynaptic $\mathrm{Ca}^{2+}$ influx. However, several observations, such as the increased amplitude of the fast recovery phase after pool depletion or the slowing of EPSC decay kinetics upon presynaptic

Fig. 8 Dysbindin overexpression increases release of EGTA-sensitive vesicles that are required for PHP. a Immunostaining of a larval NMJ (muscle 6/7) overexpressing venus-dysbindin presynaptically. Venus-Dysbindin was detected with anti-GFP (aGFP), neuronal membrane with an antibody against horseradish peroxidase (aHRP); scale bar $=5 \mu \mathrm{m}$. b Representative EPSCs of controls (elav ${ }^{C 155}$ ) and after presynaptic venus-dysbindin overexpression (elav ${ }^{C 155}$-Gal4/Y;i,UAS-venus-dysbindin/+; 'dysb oe') at $1 \mathrm{mM}\left[\mathrm{Ca}^{2+}\right]_{\mathrm{e}}$. c Quantification of EPSC amplitude/charge of the experiment shown in (b). dysbindin overexpression increases EPSC amplitude and charge compared to controls. Mean \pm s.e.m.; $n \geq 14$ cells; ${ }^{*} p<0.05$; ${ }^{* *} p<0.001$; Student's $t$-test. $\mathbf{d}$ Representative EPSC trains in response to $60-\mathrm{Hz}$ stimulation (60 stimuli, $1 \mathrm{mM}\left[\mathrm{Ca}^{2+}\right]_{\mathrm{e}}$ ) for controls and venus-dysbindin overexpressing NMJs. e Cumulative EPSC charge was calculated by back-extrapolation of a linear fit (black line) to the last 15 stimuli of the cumulative EPSC integrals to time point zero. Mean \pm s.e.m.; $n \geq 10$ cells; ${ }^{*} p<0.05$; Student's $t$-test. $\mathbf{f}$ Representative EPSCs after presynaptic venus-dysbindin overexpression and application of 50 $\mu$ M EGTA-AM or DMSO for $10 \mathrm{~min}\left(1 \mathrm{mM}\left[\mathrm{Ca}^{2+}\right]_{\mathrm{e}}\right)$. $\mathbf{g}$ Quantification of EPSC amplitude/charge of the experiment shown in (f). Application of EGTA-AM significantly decreased EPSC amplitude/charge, suggesting that dysbindin overexpression enhances the recruitment of EGTA-sensitive vesicles. Mean \pm s.e. m.; $n \geq 18$ cells; ${ }^{\star \star} p<0.001 ;{ }^{\star \star \star \star} p<0.00001$; Student's $t$-test. $\mathbf{h}$ Representative EPSCs of wild-type $\left(w^{1118}\right)$ and dysbindin mutants under baseline conditions and after treatment with PhTX or PhTX+EGTA-AM $\left(1 \mathrm{mM}\left[\mathrm{Ca}^{2+}\right]_{\mathrm{e}}\right)$. i Quantification of EPSC amplitude/charge of the experiment shown in (h). EPSC amplitudes of dysbindin mutants are similar to wild-type under baseline conditions, but decreased after PhTX application, indicating a defect in PHP. Additional treatment with EGTA-AM does not further reduce EPSC amplitude/charge. j Summary and model: Top: Glutamate receptor perturbation or proteasome perturbation induce an increase in presynaptic $\mathrm{Ca}^{2+}$ influx ${ }^{40}$ and enhance release of EGTA-sensitive, low- $p_{r}$ vesicles. Proteasome perturbation blocks PHP. Our data suggest that proteasome function opposes the release of EGTA-sensitive vesicles (black 'T') that are potentiated during homeostatic plasticity. Bottom: dysbindin mutants fail to increase release upon glutamate receptor perturbation ${ }^{11}$ or proteasome perturbation, implying that dysbindin controls the release of EGTA-sensitive vesicles 
proteasome perturbation, which are not seen after increasing $\left[\mathrm{Ca}^{2}\right.$ $\left.{ }^{+}\right]_{e}$, indicate that the increase in release is not caused by presynaptic $\mathrm{Ca}^{2+}$ influx alone. Together, these data imply that a combination of increased presynaptic $\mathrm{Ca}^{2+}$ influx and RRP size underlie the enhancement of release after proteasome or glutamate receptor perturbation. Which molecular mechanisms may link UPS function to the modulation of presynaptic $\mathrm{Ca}^{2+}$ influx or RRP size? Genetic data suggest that dysbindin functions independently of presynaptic $\mathrm{Ca}^{2+}$ influx ${ }^{11}$. Interestingly, rim mutants were shown to have a defect in homeostatic RRP size modulation, but unchanged homeostatic control of presynaptic $\mathrm{Ca}^{2+}$ influx ${ }^{15}$. We therefore speculate that RIM and Dysbindin may be involved in proteasome-dependent RRP size regulation.

How do our observations relate to mammalian synapses? At cultured hippocampal rat synapses, proteasome inhibition augments recycling vesicle pool size ${ }^{29}$ or prevents a decrease in RRP size induced by prolonged $(4 \mathrm{~h})$ depolarization ${ }^{25}$. Moreover, several studies at mammalian synapses suggest that UPSdependent regulation of RIM abundance regulates neurotransmitter release during baseline synaptic transmission and synaptic plasticity $25,26,30$. Finally, there is evidence that ubiquitination acutely regulates release on the minute time scale at cultured hippocampal rat synapses ${ }^{27}$. Together, these studies suggest that rapid, UPS-dependent control of RRP size may be evolutionarily conserved. Future studies will further elucidate the molecular signaling pathways relating UPS function to neurotransmitter release during baseline synaptic transmission and homeostatic plasticity.

\section{Methods}

Fly stocks and genetics. Drosophila stocks were maintained at $21^{\circ} \mathrm{C}-25^{\circ} \mathrm{C}$ on normal food. The $w^{1118}$ strain was used as a wild-type control. The GluRIIA ${ }^{\text {SP16 }}$ null mutation ${ }^{10}$, rim103 $153^{15}$, rbpSTOP1, rbpS2.0157, rab3-GAP ${ }^{14}$, dysbindin ${ }^{11}$, snapin $\mathrm{RNAi}^{21}, c a c^{s}, \operatorname{exn}^{E Y 10953 ; 13}$ and $p p k 16^{166 ; 18}$ were a kind gift from the lab of Graeme Davis. Cut-up ${ }^{36}$ (ctp) mutant flies were a kind gift from Benjamin Eaton, and $f i f e^{58}$ mutants were kindly provided by Kate O'Connor-Giles. For pan-neuronal

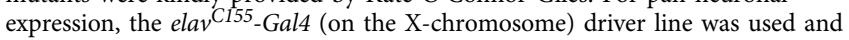
male larvae were recorded. For expression in muscle cells, the $24 \mathrm{~B}$-Gal4 driver line was used. Both driver lines, as well as the UAS-DTS5 $\left(\operatorname{Pros} \beta 6^{35}\right)$ and the UASDTS5;DTS7 (Pros $\beta 6$; Pros $\beta 2^{35}$ ) lines, were obtained from Bloomington Drosophila Stock Center (Bloomington, IN, USA). One or two DTS copies were overexpressed at a permissive temperature of $25^{\circ} \mathrm{C}$ throughout development ${ }^{35}$. To obtain elav; GluRIIA $^{\text {SP16 }}$, DTS5 flies, GluRIIA ${ }^{\text {SP16 }}$ mutant flies were recombined on the second chromosome with UAS-DTS5 flies and crossed with elav ${ }^{C 155}$-Gal4 for a stable expression of UAS-DTS5 in the GluRIIA ${ }^{S P 16}$ mutant background. Standard second and third chromosome balancer lines (Bloomington) and genetic strategies were used for all crosses and for maintaining mutant lines. For the generation of the transgenic venus-dysbindin overexpressing flies (Fig. 8), constructs based on the $p U A S T$-attB-FLAG vector backbone were injected into the $Z P$-attP-86Fb fly line harboring a landing site on the third chromosome according to standard procedures $^{59}$.

Electrophysiology. Electrophysiological recordings were made from third-instar larvae at the wandering stage. Larvae were dissected and sharp-electrode recordings were made from muscle 6 in abdominal segments 2 and 3 using an Axoclamp 900A amplifier (Molecular Devices) or an EPC 10/2 amplifier (HEKA) ${ }^{9}$. The extracellular HL3 saline contained (in mM): $70 \mathrm{NaCl}, 5 \mathrm{KCl}, 10 \mathrm{MgCl}_{2}, 10 \mathrm{NaHCO}_{3}$, 115 sucrose, 5 trehalose, 5 HEPES, $0.3 \mathrm{CaCl}_{2}$ for single-electrode recordings (Figs. 1 and 2; for Fig. 7 see figure legend) or $1 \mathrm{CaCl}_{2}$ for TEVC recordings and $\mathrm{Ca}^{2+}$ imaging experiments (Figs 4, 5, 6 and 8). To induce PHP, larvae were incubated in $20 \mu \mathrm{M}$ Philanthotoxin-433 (Sigma-Aldrich, P207) (to induce a reliable effect for TEVC recordings, $30 \mu \mathrm{M}$ PhTX was used) for $10 \mathrm{~min}$ at room temperature. For proteasome inhibition, larvae were incubated in $100 \mu \mathrm{M}$ lactacystin (SigmaAldrich, L6785) for 15-45 min (see figure legends) at room temperature (for Supplementary Fig. 1, concentration and incubation time as indicated) or $50 \mu \mathrm{M}$ MG-132 (Sigma-Aldrich, SML1135). After PhTX and lactacystin treatment, larvae were washed with HL3, dissected and recordings were carried out.

For EGTA-AM treatment, larvae were dissected before treatment, incubated with $50 \mu \mathrm{M}$ EGTA-AM (Molecular Probes, E1219) for $10 \mathrm{~min}$ at room temperature and washed thoroughly with HL3, before recordings were carried out.

From each muscle cell, 30 AP-evoked EPSPs were recorded (stimulus duration, $1 \mathrm{~ms}$ ) and averaged. At least $100 \mathrm{mEPSPs}$ were recorded and averaged for each cell to obtain the average mEPSP amplitude. Quantal content was calculated by dividing the average EPSP amplitude by the average mEPSP amplitude for each cell.

TEVC recordings were performed using an Axoclamp 900A Amplifier (Molecular Devices). Cells were clamped to a membrane potential of $-60 \mathrm{mV}$. A total of 50 EPSCs were averaged to obtain the mean EPSC (stimulus duration, 1 ms) value for each cell. AP-evoked EPSCs were recorded with a combination of a HS-9A $\times 10$ and a HS-9A $\times 1$ headstage (Molecular Devices), whereas mEPSC recordings were carried out using two HS-9A $\times 1$ headstages (Axon CNS, Molecular Devices). The size of the RRP of synaptic vesicles was calculated by the method of cumulative EPSC charge ${ }^{32,43}$. Synapses were stimulated with $60 \mathrm{~Hz}$ trains (60 stimuli, 5 trains per cell). The cumulative EPSC charge was obtained by backextrapolating a linear fit to the last 15 cumulative EPSC charge values of the $60 \mathrm{~Hz}$ train to time zero. In cases where EGTA-AM had been applied (Supplementary Fig. 5), cumulative EPSC amplitude was calculated with the Elmqvist and Quastel method $^{60,61}$. Here, a linear fit to the first two points of a plot of peak EPSC amplitude versus cumulative EPSC amplitude was extrapolated to the abscissa. This extrapolation gave the cumulative EPSC amplitude for the RRP size estimate. For the recovery experiments, synapses were stimulated with a $60 \mathrm{~Hz}$ train for $1 \mathrm{~s}(2$ $\mathrm{mM}\left[\mathrm{Ca}^{2+}\right]_{\mathrm{e}}$ ) followed by single stimuli applied at $0.025 \mathrm{~s}, 0.075 \mathrm{~s}, 0.175 \mathrm{~s}, 0.475 \mathrm{~s}$, $1.475 \mathrm{~s}, 4.475 \mathrm{~s}$ and $10.475 \mathrm{~s}$ after the train. Average EPSC amplitudes during recovery were normalized to the first EPSC amplitude of the $60 \mathrm{~Hz}$ train and fitted with a double-exponential constrained to an amplitude of one.

$\mathbf{C a}^{2+}$ imaging. Wandering third-instar larvae were dissected in $\mathrm{Ca}^{2+}$-free HL3, leaving brain and nerves intact ${ }^{45}$. Nerves were cut close to the NMJ in the presence of $\mathrm{Ca}^{2+}$-free HL3 containing 5.6 mM Oregon Green 488 Bapta-1 and $1 \mathrm{mM}$ Alexa568 (both from Invitrogen). Thereafter, the preparation was put on ice for $5 \mathrm{~min}$ and washed thoroughly for another $5 \mathrm{~min}$. After changing the extracellular solution to HL3 containing $1 \mathrm{mM} \mathrm{Ca}{ }^{2+}$, spatially averaged $\mathrm{Ca}^{2+}$ transients in response to single-AP stimulation (stimulus duration, $3 \mathrm{~ms}$ ) were imaged in type $1 \mathrm{~b}$ boutons synapsing onto muscle 6/7 of abdominal segments A2/A3 with a two-photon microscope (Scientifica). The $780 \mathrm{~nm}$ excitation light of a Mai Tai laser (Spectra Physics) was focused onto individual boutons with a $40 \times$ water-dipping objective (Olympus, 1.0 NA), and line scans across single boutons were made at $333 \mathrm{~Hz}$ for $1 \mathrm{~s}$. The average fluorescence change per bouton is based on 10 sweeps (inter-sweep interval, $10 \mathrm{~s}$ ). Traces were background subtracted and corrected for bleaching using an exponential fit. If the transient increase in OGB-1 fluorescence could not properly be fit with a single exponential, the $\tau$ value was excluded from analysis. Changes in fluorescence were quantified as $\Delta G / R=\left(G_{\text {peak }}-G_{\text {base }}\right) / R$, where $G_{\text {peak }}$ is the peak fluorescence intensity in response to stimulation, and $G_{\text {base }}$ and $R$ are the average fluorescence intensity during $150 \mathrm{~ms}$ before stimulation in the green and red channel, respectively.

Data analysis. Electrophysiology data were acquired with Clampex (Axon CNS, Molecular Devices) or PatchMaster (HEKA) and analyzed with custom-written routines in Igor Pro (Wavemetrics). Spontaneous mEPSPs and mEPSCs were analyzed with Mini Analysis Software (Synaptosoft). Average mEPSC waveforms were obtained with AxoGraph X. $\mathrm{Ca}^{2+}$ imaging data were acquired with Helio-Scan ${ }^{62}$ and analyzed with custom-written Igor Pro routines. Statistical analysis was done with Prism software (GraphPad software). Data were tested for normality using D'Agostino-Pearson omnibus normality test. Normally distributed data were analyzed for statistical differences via $t$-test (pairwise comparison) or analysis of variance (ANOVA) and Tukey's test for multiple comparisons. For non-normally distributed data, Wilcoxon rank-sum test or Dunn's multiple comparisons after nonparametric ANOVA were used. Data are given as mean \pm s.e.m.

Immunohistochemistry. Third-instar larval preparations were fixed for $2 \mathrm{~min}$ with Bouin's fixative (100\%, Sigma-Aldrich, HT-10132), or 100\% ice-cold Ethanol for 5 min. For proteasome blockade, larvae were incubated with $100 \mu \mathrm{M}$ lactacystin for 15,30 or $45 \mathrm{~min}$ at room temperature before applying the fixative. Control larvae were incubated with HL3 saline for the same time durations. Preparations were washed thoroughly with PBS containing $0.05 \%$ Triton X-100. After washing, preparations were blocked with $3 \%$ normal goat serum in PBS containing $0.05 \%$ Triton X-100. Incubation with the primary antibody was done at $4{ }^{\circ} \mathrm{C}$ on a rotating platform overnight. The following primary antibodies and dilutions were used in this study: anti-ubiquitin (FK2, mouse, Calbiochem ST1200; 1:5000), anti-discs large (rabbit, DSHB AB_2617529; 1:10,000), anti-Bruchpilot (mouse, nc82; 1:100), anti-GFP (rabbit, Life Technologies G10362; 1:1000) and anti-HRP Alexa-Fluor 647 (goat, Jackson ImmunoResearch 123-605-021; 1:200).

Secondary antibodies (Life Technologies; 1:500) were applied for $2 \mathrm{~h}$ at room temperature on a rotating platform. Preparations were mounted onto slides with ProLong Gold (Life Technologies, P36930). Images were acquired on an LSM710 confocal microscope (Carl Zeiss), using Zen software (Carl Zeiss). Image analysis was carried out in ImageJ. Quantification of fluorescence intensity was done on maximal projections. Figures were assembled using Adobe Photoshop and Illustrator software.

Data availability. The data that support the findings of this study are available from the corresponding author upon reasonable request. 
Received: 27 April 2017 Accepted: 1 December 2017

Published online: 18 January 2018

\section{References}

1. Cohen, L. D. et al. Metabolic turnover of synaptic proteins: kinetics, interdependencies and implications for synaptic maintenance. PLoS. ONE 8, e63191 (2013)

2. Djakovic, S. N., Schwarz, L. A., Barylko, B., DeMartino, G. N. \& Patrick, G. N. Regulation of the proteasome by neuronal activity and calcium/calmodulindependent protein kinase II. J. Biol. Chem. 284, 26655-26665 (2009).

3. Speese, S. D., Trotta, N., Rodesch, C. K., Aravamudan, B. \& Broadie, K. The ubiquitin proteasome system acutely regulates presynaptic protein turnover and synaptic efficacy. Curr. Biol. 13, 899-910 (2003).

4. Davis, G. W. Homeostatic signaling and the stabilization of neural function. Neuron 80, 718-728 (2013).

5. Marder, E. \& Goaillard, J.-M. Variability, compensation and homeostasis in neuron and network function. Nat. Rev. Neurosci. 7, 563-574 (2006).

6. Pozo, K. \& Goda, Y. Unraveling mechanisms of homeostatic synaptic plasticity. Neuron 66, 337-351 (2010).

7. Turrigiano, G. Homeostatic synaptic plasticity: local and global mechanisms for stabilizing neuronal function. Cold Spring Harb. Perspect. Biol. 4, a005736 (2012).

8. Davis, G. W. \& Müller, M. Homeostatic control of presynaptic neurotransmitter release. Annu. Rev. Physiol. 77, 251-270 (2015).

9. Davis, G. W., DiAntonio, A., Petersen, S. A. \& Goodman, C. S. Postsynaptic PKA controls quantal size and reveals a retrograde signal that regulates presynaptic transmitter release in Drosophila. Neuron 20, 305-315 (1998).

10. Petersen, S. A., Fetter, R. D., Noordermeer, J. N., Goodman, C. S. \& DiAntonio, A. Genetic analysis of glutamate receptors in Drosophila reveals a retrograde signal regulating presynaptic transmitter release. Neuron 19, 1237-1248 (1997).

11. Dickman, D. K. \& Davis, G. W. The schizophrenia susceptibility gene dysbindin controls synaptic homeostasis. Science 326, 1127-1130 (2009).

12. Frank, C. A., Kennedy, M. J., Goold, C. P., Marek, K. W. \& Davis, G. W. Mechanisms underlying the rapid induction and sustained expression of synaptic homeostasis. Neuron 52, 663-677 (2006).

13. Frank, C. A., Pielage, J. \& Davis, G. W. A presynaptic homeostatic signaling system composed of the Eph receptor, ephexin, Cdc42, and CaV2.1 calcium channels. Neuron 61, 556-569 (2009).

14. Müller, M., Pym, E. C. G., Tong, A. \& Davis, G. W. Rab3-GAP controls the progression of synaptic homeostasis at a late stage of vesicle release. Neuron 69 , 749-762 (2011).

15. Müller, M., Liu, K. S. Y., Sigrist, S. J. \& Davis, G. W. RIM controls homeostatic plasticity through modulation of the readily-releasable vesicle pool. J. Neurosci. 32, 16574-16585 (2012).

16. Müller, M., Genç, Ö. \& Davis, G. W. RIM-binding protein links synaptic homeostasis to the stabilization and replenishment of high release probability vesicles. Neuron 85, 1056-1069 (2015).

17. Wang, T., Hauswirth, A. G., Tong, A., Dickman, D. K. \& Davis, G. W. Endostatin is a trans-synaptic signal for homeostatic synaptic plasticity. Neuron 83, 616-629 (2014)

18. Younger, M. A., Müller, M., Tong, A., Pym, E. C. \& Davis, G. W. A presynaptic ENaC channel drives homeostatic plasticity. Neuron 79, 1183-1196 (2013).

19. Talbot, K. et al. Dysbindin-1 is reduced in intrinsic, glutamatergic terminals of the hippocampal formation in schizophrenia. J. Clin. Invest. 113, 1353-1363 (2004).

20. Weickert, C. S., Rothmond, D. A., Hyde, T. M., Kleinman, J. E. \& Straub, R. E. Reduced DTNBP1 (dysbindin-1) mRNA in the hippocampal formation of schizophrenia patients. Schizophr. Res. 98, 105-110 (2008).

21. Dickman, D. K., Tong, A. \& Davis, G. W. Snapin is critical for presynaptic homeostatic plasticity. J. Neurosci. 32, 8716-8724 (2012).

22. Mullin, A. P. et al. Gene dosage in the dysbindin schizophrenia susceptibility network differentially affect synaptic function and plasticity. J. Neurosci. 35, 325-338 (2015).

23. Gokhale, A. et al. The proteome of BLOC-1 genetic defects identifies the Arp $2 / 3$ actin polymerization complex to function downstream of the schizophrenia susceptibility factor dysbindin at the synapse. J. Neurosci. 36, 12393-12411 (2016).

24. Hegde, A. N. The ubiquitin-proteasome pathway and synaptic plasticity. Learn. Mem. 17, 314-327 (2010).

25. Jiang, X. et al. A role for the ubiquitin-proteasome system in activity-dependent presynaptic silencing. J. Neurosci. 30, 1798-1809 (2010).

26. Lazarevic, V., Schone, C., Heine, M., Gundelfinger, E. D. \& Fejtova, A Extensive remodeling of the presynaptic cytomatrix upon homeostatic adaptation to network activity silencing. J. Neurosci. 31, 10189-10200 (2011).
27. Rinetti, G. V. \& Schweizer, F. E. Ubiquitination acutely regulates presynaptic neurotransmitter release in mammalian neurons. J. Neurosci. 30, 3157-3166 (2010).

28. Waites, C. L. et al. Bassoon and Piccolo maintain synapse integrity by regulating protein ubiquitination and degradation. EMBO J. 32, 954-969 (2013).

29. Willeumier, K., Pulst, S. M. \& Schweizer, F. E. Proteasome inhibition triggers activity-dependent increase in the size of the recycling vesicle pool in cultured hippocampal neurons. J. Neurosci. 26, 11333-11341 (2006).

30. Yao, I. et al. SCRAPPER-dependent ubiquitination of active zone protein RIM1 regulates synaptic vesicle release. Cell 130, 943-957 (2007).

31. Eggermann, E., Bucurenciu, I., Goswami, S. P. \& Jonas, P. Nanodomain coupling between $\mathrm{Ca}^{2+}$ channels and sensors of exocytosis at fast mammalian synapses. Nat. Rev. Neurosci. 13, 7-21 (2012).

32. Neher, E. Merits and limitations of vesicle pool models in view of heterogeneous populations of synaptic vesicles. Neuron 87, 1131-1142 (2015).

33. Böhme, M. A. et al. Active zone scaffolds differentially accumulate Unc13 isoforms to tune $\mathrm{Ca}^{2+}$ channel-vesicle coupling. Nat. Neurosci. 19, 1311-1320 (2016).

34. Reddy-Alla, S. et al Stable positioning of Unc13 restricts synaptic vesicle fusion to defined release sites to promote synchronous neurotransmission. Neuron $\mathbf{9 5}$, 1350-1364.e12 (2017).

35. Smyth, K. A. \& Belote, J. M. The dominant temperature-sensitive lethal DTS7 of Drosophila melanogaster encodes an altered 20S proteasome $\beta$-type subunit. Genetics 151, 211-220 (1999).

36. Kreko-Pierce, T. \& Eaton, B. A. The Drosophila LC8 homologue Cut-up specifies the axonal transport of proteasomes. J. Cell Sci. 130, 3388-3398 (2017).

37. Bingol, B. \& Schuman, E. M. Activity-dependent dynamics and sequestration of proteasomes in dendritic spines. Nature 441, 1144-1148 (2006).

38. Jan, L. Y. \& Jan, Y. N. Antibodies to horseradish peroxidase as specific neuronal markers in Drosophila and in grasshopper embryos. Proc. Natl. Acad. Sci. USA 79, 2700-2704 (1982).

39. Jan, L. Y. \& Jan, Y. N. Properties of the larval neuromuscular junction in Drosophila melanogaster. J. Physiol. 262, 189-214 (1976).

40. McEwen, J. M., Madison, J. M., Dybbs, M. \& Kaplan, J. M. Antagonistic regulation of synaptic vesicle priming by tomosyn and UNC-13. Neuron 51, 303-315 (2006).

41. Vyleta, N. P. \& Jonas, P. Loose coupling between $\mathrm{Ca}^{2+}$ channels and release sensors at a plastic hippocampal synapse. Science 343, 665-670 (2014).

42. Smith, P. D., Liesegang, G. W., Berger, R. L., Czerlinski, G. \& Podolsky, R. J. A stopped-flow investigation of calcium ion binding by ethylene glycol bis( $\beta$ aminoethyl ether)-N,N'-tetraacetic acid. Anal. Biochem. 143, 188-195 (1984).

43. Schneggenburger, R., Meyer, A. C. \& Neher, E. Released fraction and total size of a pool of immediately available transmitter quanta at a calyx synapse. Neuron 23, 399-409 (1999).

44. Weyhersmüller, A., Hallermann, S., Wagner, N. \& Eilers, J. Rapid active zone remodeling during synaptic plasticity. J. Neurosci. 31, 6041-6052 (2011).

45. Müller, M. \& Davis, G. W. Transsynaptic control of presynaptic $\mathrm{Ca}^{2+}$ influx achieves homeostatic potentiation of neurotransmitter release. Curr. Biol. 22, 1102-1108 (2012).

46. Hallermann, S. \& Silver, R. A. Sustaining rapid vesicular release at active zones: potential roles for vesicle tethering. Trends Neurosci. 36, 185-194 (2013).

47. Sakaba, T. \& Neher, E. Calmodulin mediates rapid recruitment of fast-releasing synaptic vesicles at a Calyx-type synapse. Neuron 32, 1119-1131 (2001).

48. Wu, L.-G. \& Borst, J. G. G. The reduced release probability of releasable vesicles during recovery from short-term synaptic depression. Neuron 23, 821-832 (1999).

49. Frank, C. A. Homeostatic plasticity at the Drosophila neuromuscular junction. Neuropharmacology 78, 63-74 (2013).

50. Ghiani, C. A. \& Dell'Angelica, E. C. Dysbindin-containing complexes and their proposed functions in brain: from zero to (too) many in a decade. ASN Neuro 3, 109-124 (2011).

51. Ding, W.-X. et al. Linking of autophagy to ubiquitin-proteasome system is important for the regulation of endoplasmic reticulum stress and cell viability. Am. J. Pathol. 171, 513-524 (2007).

52. Hakim, V., Cohen, L. D., Zuchman, R. Ziv, T. \& Ziv, N. E. The effects of proteasomal inhibition on synaptic proteostasis. EMBO J. 35, 2238-2262 (2016).

53. Shen, Y., Tang, Y., Zhang, X., Huang, K. \& Le, W. Adaptive changes in autophagy after UPS impairment in Parkinson's disease. Acta Pharmacol. Sin. 34, 667-673 (2013)

54. Hu, Z., Tong, X.-J. \& Kaplan, J. M. UNC-13L, UNC-13S, and Tomosyn form a protein code for fast and slow neurotransmitter release in Caenorhabditis elegans. eLife 2, e00967 (2013).

55. Wang, X., Pinter, M. J. \& Rich, M. M. Reversible recruitment of a homeostatic reserve pool of synaptic vesicles underlies rapid homeostatic plasticity of quantal content. J. Neurosci. 36, 828-836 (2016). 
56. Thanawala, M. S. \& Regehr, W. G. Presynaptic calcium influx controls neurotransmitter release in part by regulating the effective size of the readily releasable pool. J. Neurosci. 33, 4625-4633 (2013).

57. Liu, K. S. Y. et al. RIM-binding protein, a central part of the active zone, is essential for neurotransmitter release. Science 334, 1565-1569 (2011).

58. Bruckner, J. J. et al. Fife, a Drosophila Piccolo-RIM homolog, promotes active zone organization and neurotransmitter release. J. Neurosci. 32, 17048-17058 (2012).

59. Bischof, J., Sheils, E. M., Björklund, M. \& Basler, K. Generation of a transgenic ORFeome library in Drosophila. Nat. Protoc. 9, 1607-1620 (2014).

60. Elmqvist, D. \& Quastel, D. M. A quantitative study of end-plate potentials in isolated human muscle. J. Physiol. 178, 505-529 (1965).

61. Thanawala, M. S. \& Regehr, W. G. Determining synaptic parameters using high-frequency activation. J. Neurosci. Methods 264, 136-152 (2016).

62. Langer, D. et al. HelioScan: a software framework for controlling in vivo microscopy setups with high hardware flexibility, functional diversity and extendibility. J. Neurosci. Methods 215, 38-52 (2013).

\section{Acknowledgements}

This research was funded by a 'Forschungskredit' grant of the University of Zurich to C. W. and a Swiss National Science Foundation Assistant Professor grant (PP00P3-15) to M.M. We thank Philipp Bethge and Fritjof Helmchen for sharing the $2 \mathrm{P}$ set-up and for providing technical support. Moreover, we thank Marc Debrunner for Drosophila embryo injections. We are grateful to Grae Davis, Dion Dickman and members of the Müller lab for helpful discussions and critical comments on the manuscript.

\section{Author contributions}

M.M. and C.W. conceptualized and designed experiments. C.W., I.D., S.S., O.G. and M.M. conducted research, analyzed and interpreted data. C.W. and M.M. wrote the manuscript.

\section{Additional information}

Supplementary Information accompanies this paper at https://doi.org/10.1038/s41467017-02494-0.

Competing interests: The authors declare no competing financial interests.

Reprints and permission information is available online at http://npg.nature.com/ reprintsandpermissions/

Publisher's note: Springer Nature remains neutral with regard to jurisdictional claims in published maps and institutional affiliations.

(c) (i) Open Access This article is licensed under a Creative Commons Attribution 4.0 International License, which permits use, sharing, adaptation, distribution and reproduction in any medium or format, as long as you give appropriate credit to the original author(s) and the source, provide a link to the Creative Commons license, and indicate if changes were made. The images or other third party material in this article are included in the article's Creative Commons license, unless indicated otherwise in a credit line to the material. If material is not included in the article's Creative Commons license and your intended use is not permitted by statutory regulation or exceeds the permitted use, you will need to obtain permission directly from the copyright holder. To view a copy of this license, visit http://creativecommons.org/ licenses/by/4.0/.

(C) The Author(s) 2018 